\title{
ANCHORED EXPANSION, SPEED AND THE POISSON-VORONOI TESSELLATION IN SYMMETRIC SPACES
}

\author{
By Itai Benjamini, Elliot Paquette ${ }^{1}$ and Joshua Pfeffer \\ The Weizmann Institute of Science, The Ohio State University and \\ Massachusetts Institute of Technology
}

\begin{abstract}
We show that a random walk on a stationary random graph with positive anchored expansion and exponential volume growth has positive speed. We also show that two families of random triangulations of the hyperbolic plane, the hyperbolic Poisson-Voronoi tessellation and the hyperbolic PoissonDelaunay triangulation, have 1-skeletons with positive anchored expansion. As a consequence, we show that the simple random walks on these graphs have positive hyperbolic speed. Finally, we include a section of open problems and conjectures on the topics of stationary geometric random graphs and the hyperbolic Poisson-Voronoi tessellation.
\end{abstract}

1. Introduction. A rooted, locally finite, unlabeled random graph $(G, \rho)$ is called stationary if the distribution of $(G, \rho)$ is the same as the distribution of $\left(G, X_{1}\right)$ where $X_{1}$ is a simple random walk on $G$ started from $\rho$ after 1 step. Such a graph is called reversible if in addition the birooted equivalence class $\left(G, X_{0}, X_{1}\right)$ has the same distribution as $\left(G, X_{1}, X_{0}\right)$. Stationary random graphs enjoy many of the same properties of transitive graphs, which they generalize. For example, in [8] it is shown that a stationary random graph of subexponential growth is almost surely Liouville. In [16], the converse is shown under the additional assumption of ergodicity.

The property of reversibility is closely tied to the more familiar notion of unimodularity. Let $P$ be the law of a unimodular random graph $(G, \rho)$ for which $\mathbb{E} \operatorname{deg}(\rho)<\infty$. Let $Q$ be the law on rooted graphs which is absolutely continuous to $P$ and has the Radon-Nikodym derivative $\frac{d Q}{d P}=\frac{\operatorname{deg}(\rho)}{\mathbb{E} \operatorname{deg}(\rho)}$. Then it can be checked that $P$ is unimodular if and only if $Q$ is reversible (see [8], Proposition 2.5). Hence, statements that hold almost surely for $P$ hold almost surely for $Q$, and provided the degree of $\rho$ is almost surely positive, statements that hold almost surely for $Q$ hold almost surely for $P$.

For a finite subset of vertices $S \subseteq \mathcal{V}(G)$, let $\operatorname{Vol}_{G}(S)$ be the sum of degrees of the vertices of $S$, and let $|\partial S|$ denote the number of edges that have exactly

Received October 2014; revised July 2017.

${ }^{1}$ Supported by NSF Postdoctoral Fellowship DMS-1304057.

MSC2010 subject classifications. Primary 60D05; secondary 60G55, 52C20.

Key words and phrases. Hyperbolic space, hyperbolic geometry, Poisson process, Voronoi tiling, tessellation, isoperimetric constant, anchored isoperimetric constant, expansion, unimodular random graph. 
one terminus in $S$. A locally finite connected, rooted graph $(G, \rho)$ is said to have positive anchored expansion if

$$
i^{*}(G):=\liminf _{\substack{\left.|S| \rightarrow \infty \\ \rho \in S \\ G\right|_{S} \text { connected }}} \frac{|\partial S|}{\operatorname{Vol}_{G}(S)}>0 .
$$

Note that, in a connected graph, $i^{*}(G)$ is independent of the root chosen. This definition is a natural relaxation of the condition of having a positive edge isoperimetric constant, in which the liminf is replaced by the infimum over all finite $S$.

There are many examples of random graph processes that fail to have a positive edge isoperimetric constant but do have positive anchored expansion, such as supercritical Galton-Watson trees conditioned on nonextinction [32]. A key feature of positive anchored expansion is that it is stable under random perturbations: for $p$ sufficiently large, $p$-Bernoulli bond or site percolation on a graph with positive anchored expansion gives a graph with positive anchored expansion $[10,11$, 17].

There are perhaps three fundamental known consequences of positive anchored expansion. The earliest follows from Thomassen [35], who gives a general criterion on the isoperimetric profile of a graph that implies that simple random walk is transient; as a special case, his result shows that positive anchored expansion implies that simple random walk is transient. The second, due to Virág [36], is that under the additional assumption of bounded degree, simple random walk almost surely has positive liminf speed, that is,

$$
\liminf _{k \rightarrow \infty} \frac{d\left(\rho, X_{k}\right)}{k}>0,
$$

where $d$ is the graph metric. The third result, also due to Virág [36], is a heat kernel bound that states that, for every vertex $x$, there is an $N$ so that for all $n>N$ and all vertices $y$,

$$
p^{n}(x, y)<e^{-\alpha n^{1 / 3}} .
$$

Further consequences of positive anchored expansion, such as the existence of a phase transition in the Ising model with nonzero external field are shown in [25].

We will show that a stationary graph with positive anchored expansion and exponential growth has positive speed, thus effectively trading the bounded degree assumption of [36] for the assumptions of stationarity and exponential growth.

THEOREM 1.1. Let $\delta$ be a pseudometric on $G$ that is stationary in the sense that $\left\{\left(G, X_{k}, \delta\right)\right\}_{0}^{\infty}$ is a stationary sequence of pseudometric graphs. Suppose that:

(1) $\mathbb{E} \delta\left(\rho, X_{1}\right)<\infty$

(2) $(G, \rho)$ has positive anchored expansion almost surely. 
(3) With $B_{\delta}(\rho, r)$ denote the closed ball of radius $r$ around $\rho$,

$$
\liminf _{r \rightarrow \infty}\left|B_{\delta}(\rho, r)\right|^{1 / r}<\infty
$$

almost surely.

Then simple random walk $X_{k}$ started from $\rho$ has positive speed, that is, the limit

$$
s=\lim _{k \rightarrow \infty} \frac{\delta\left(\rho, X_{k}\right)}{k}
$$

exists and $s>0$ almost surely.

Note that further assumptions are needed to say that $s$ is nonrandom.

For certain classes of graphs, positive speed is enough to ensure the existence of nonconstant bounded harmonic functions (e.g., planar, bounded degree graphs [12]). We show that a stationary random graph with nonconstant bounded harmonic functions must have an infinite-dimensional space of such functions.

THEOREM 1.2. A stationary random graph $(G, \rho)$ either has a 1-dimensional space of bounded harmonic functions or an infinite-dimensional space of bounded harmonic functions.

Work of [16] shows that under mild conditions on a stationary graph, positive speed implies the existence of an infinite-dimensional space of bounded harmonic functions. Hence, both $\mathscr{D}^{\lambda}$ and $\mathscr{V}^{\lambda}$ have infinite spaces of bounded harmonic functions. Improvements to [16] made since the original draft of this paper appeared now give a direct proof that positive speed implies an infinite dimensional space of bounded harmonic functions.

Hyperbolic Poisson-Voronoi tessellation. Theorems 1.1 and 1.2 are tailormade for random graphs that arise as invariant random perturbations of homogeneous spaces, such as invariant percolation on non-amenable Cayley graphs. We will show how this framework can be applied to a random discretization of Riemannian symmetric space. Specifically, we will consider the hyperbolic plane $\mathbb{H}$.

Let $\Pi^{\lambda}$ denote a Poisson point process on $\mathbb{H}$ with intensity given by a multiple $\lambda>0$ of its invariant volume measure. The exact normalization for the area measure is given at the start of Section 3.

The hyperbolic Poisson-Voronoi tessellation of $\mathbb{H}$ is a polygonal cell complex, with each cell containing exactly one point in $\Pi^{\lambda}$; this point is referred to as the nucleus of the cell. For a point $p_{0} \in \Pi^{\lambda}$, the cell with nucleus $p_{0}$ is given by

$$
\left\{z \in \mathbb{H}: d_{\mathbb{H}}\left(z, p_{0}\right)=\min _{p \in \Pi^{\lambda}} d_{\mathbb{H}}(z, p)\right\},
$$

where $d_{\mathbb{H}}$ denotes distance in the hyperbolic metric. We denote by $\mathscr{V}^{\lambda}$ the 1 skeleton of this cell complex. 


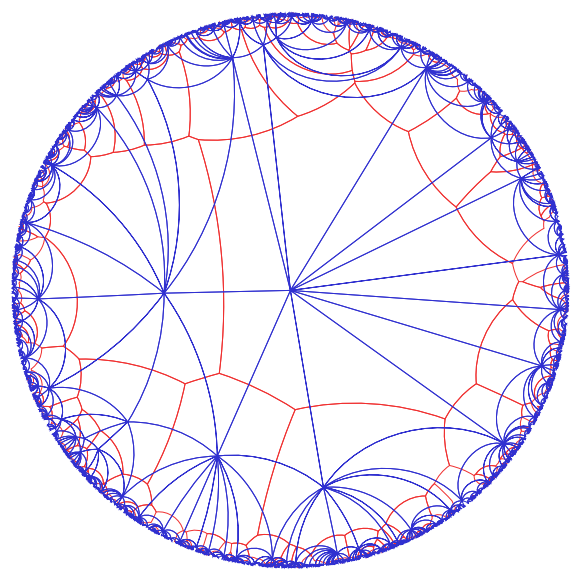

(A) $\lambda=0.2$ and $r=0.9995$.

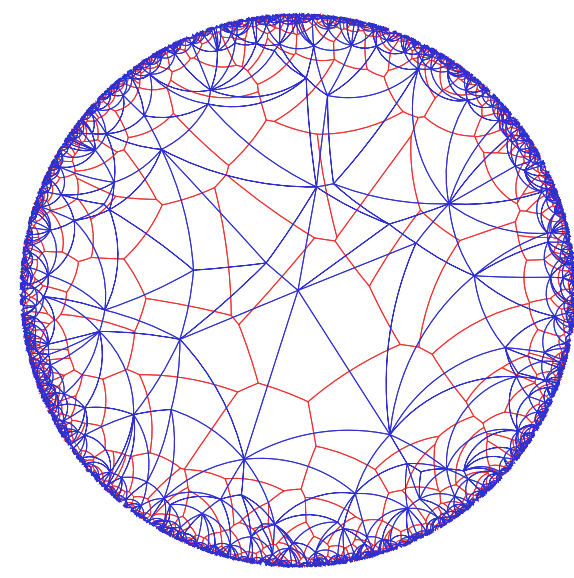

(в) $\lambda=1$ and $r=0.9975$.

FIG. 1. A rendering of the Poisson-Voronoi tessellation (red) for various $\lambda$ with superimposed Delaunay triangulation (blue). In each simulation, the process $\Pi_{r}^{\lambda}$ on $B_{\mathbb{H}}(0, r)$ is sampled with intensity $\lambda$ times hyperbolic area measure. In electronic versions of this figure, it is possible to get more detail by zooming in on the picture. Note that for smaller $\lambda$ the average degree increases.

From the point process $\Pi^{\lambda}$, we can also construct the Poisson-Delaunay complex, a polygonal cell complex with vertices $\Pi^{\lambda}$ and a hyperbolic geodesic between two vertices if and only if the corresponding Voronoi cells share a boundary edge. We denote by $\mathscr{D}^{\lambda}$ the 1 -skeleton of this cell complex. Note that $\mathscr{V}^{\lambda}$ and $\mathscr{D}^{\lambda}$ are dual graphs. (Except where absolutely necessary, we will not distinguish between the abstract graphs $\mathscr{D}^{\lambda}$ and $\mathscr{V}^{\lambda}$ and their geometric realizations as 1skeletons.) See Figure 1 for a simulation of these tessellations.

It is elementary to see that, in the Poisson-Delaunay complex, all the faces are almost surely triangles; hence, we will refer to it as the Delaunay triangulation. Further, it can be checked that a triple of points $\{x, y, z\}$ in $\Pi^{\lambda}$ forms a triangle in the Delaunay triangulation if and only if all three points lie on the boundary of a finite hyperbolic disk in $\mathbb{H}$ whose interior contains no points of $\Pi^{\lambda}$.

The probability that a disk in $\mathbb{H}$ contains no points of $\Pi^{\lambda}$ is exponentially small in the volume of the disk. As the volume of a disk centered at a fixed point in $\mathbb{H}$ grows exponentially in the radius, the maximal diameter of a Delaunay triangle incident to that point has a very thin tail.

LEMma 1.3. Let $S_{0}$ be the union of the Delaunay triangles that contains the point 0 as a vertex. There is a constant $C=C(\lambda)>0$ so that for all $r>0$

$$
\mathbb{P}\left[S_{0} \not \subset B_{\mathbb{H}}(0, r)\right] \leq C e^{r-\lambda \pi e^{r / 4}},
$$

where $B_{\mathbb{H}}(x, r)$ is the hyperbolic ball centered at $x$ of radius $r$. 
Note that, by the transitivity of the automorphism group of $\mathbb{H}$ and the invariance of the intensity of $\Pi^{\lambda}$, this lemma also holds if 0 is replaced by any other fixed point in $\mathbb{H}$ (see Remark 1.7).

Our main theorem about these tessellations is that both have positive anchored expansion almost surely.

THEOREM 1.4. For $G=\mathscr{D}^{\lambda}$ or $\mathscr{V}^{\lambda}$ there is a constant $c=c(\lambda)>0$ so that $i^{*}(G)>c$ almost surely.

REMARK 1.5. The anchored expansion constant $i^{*}(G)$ can be seen to be deterministic almost surely, by the following argument. Let $\mathcal{L}$ be a transitive tiling of the hyperbolic plane by compact polygons, transitive meaning that any polygon can be mapped to any other by a hyperbolic isometry. Each polygon has finite volume, and so almost surely each polygon contains only finitely many points. Since these points connect to only finitely many other points in both $\mathscr{D}^{\lambda}$ and $\mathscr{V}^{\lambda} i^{*}(G)$ is independent of the configurations of points in each polygon.

Enumerating the polygons, we can identify $\Pi^{\lambda}$ with

$$
\left(\Pi_{1}^{\lambda}, \Pi_{2}^{\lambda}, \Pi_{3}^{\lambda}, \ldots\right),
$$

where each $\Pi_{i}^{\lambda}$ is a Poisson point process of intensity $\lambda$ on one of the polygons, and $\Pi^{\lambda}$ is built from these point processes by isometrically mapping $\Pi_{i}^{\lambda}$ to the $i$ th polygon. From the independence of $i^{*}(G)$ from the configuration of points in any one polygon, we get that $i^{*}(G)$ is measurable with respect to the tail- $\sigma$-algebra of this space, and hence $i^{*}(G)$ is deterministic.

To these graphs, we would like to apply our general theory. Observe that $\mathscr{V}^{\lambda}$ is 3-regular. Hence by the original theorem of [36], simple random walk on $\mathscr{V}^{\lambda}$ has positive speed. Since $\mathscr{V}^{\lambda}$ is bounded degree and planar, it is also non-Liouville [12]. Provided it could be biased in such a way that it is stationary (a direction we do not pursue), it would have, by Theorem 1.2, an infinite-dimensional space of bounded harmonic functions.

As for $\mathscr{D}^{\lambda}$, which is not bounded degree, we cannot apply [36]. So we, will show the hypotheses of Theorem 1.1 are satisfied for this graph. We begin with the unimodularity of the random graph. This is a consequence of hyperbolic space having involutive isometries: at each $x \in \mathbb{H}$, there is an isometry that fixes $x$ and reverses all geodesics through $x$. A manifold which has this property is called a (global) Riemannian symmetric space. We give a brief introduction to these spaces in Section 4.

Proposition 1.6. Let $\mathbb{M}$ be a nonpositively curved Riemannian symmetric space, and fix $x \in \mathbb{M}$. Let $\mathcal{X}$ be a Poisson point process whose intensity is invariant under symmetries of $\mathbb{M}$ and which is conditioned to have a point at $x$. Denote by $\mathscr{G}$ the dual graph of the Voronoi tessellation, and let $\rho$ be the vertex of $\mathscr{G}$ embedded at $x$. Let $P$ be the distribution of $(\mathscr{G}, \rho)$. Then: 
(1) $\mathbb{E} \operatorname{deg} \rho<\infty$ and

(2) with $\frac{d Q}{d P}=\frac{\operatorname{deg} \rho}{\mathbb{E} \operatorname{deg} \rho},(\mathscr{G}, \rho)$ is reversible as an embedded random graph un$\operatorname{der} Q$.

Equivalently, $(\mathscr{G}, \rho)$ under $P$ is unimodular. We give the proof in Section 4 .

REMARK 1.7. In studying the anchored expansion properties both $\mathscr{V}^{\lambda}$ and $\mathscr{D}^{\lambda}$, we may condition on the Poisson process so that either $\mathscr{V}^{\lambda}$ and $\mathscr{D}^{\lambda}$ includes the origin as a vertex; proving Theorem 1.4 for these conditioned forms of $\mathscr{V}^{\lambda}$ and $\mathscr{D}^{\lambda}$ is equivalent to proving the theorem for the original graphs. Adding a single point to the Poisson process will modify only finitely many vertices and edges of $\mathscr{V}^{\lambda}$ and $\mathscr{D}^{\lambda}$, and the anchored expansion constant is insensitive to any such change.

Returning to the application of Theorem 1.1 to $\mathscr{D}^{\lambda}$, Proposition 1.6 gives the needed stationarity of the random graph. It further asserts that the random graph and its embedding are stationary after biasing by the degree of the root. Hence, the restriction of the hyperbolic metric to the graph is a stationary metric on the graph. The volume growth of this graph is simply the growth rate of the number of Poisson in points in concentric balls around the origin. This has exponential growth almost surely, and so random walk on $\mathscr{D}^{\lambda}$ has positive hyperbolic speed almost surely. As a consequence, in Proposition 3.13, we show that random walk on $\mathscr{D}^{\lambda}$ converges to a point on the ideal boundary of $\mathbb{H}$ (effectively that as a sequence of points in the Poincaré disk, it converges to a point on the unit circle $S^{1}$ ).

Discussion. We give a criterion for positive speed of simple random walk in terms of positive anchored expansion, which implies transience. For many classes of stationary random graphs, there is an alternative between recurrence and positive speed. For example, unimodular random trees of at most exponential growth and bounded degree planar graphs both have that random walk is almost surely recurrent or random walk has positive speed (this follows from combining results of [12] and [8]). We would expect that bounded degree unimodular Gromov hyperbolic graphs are another such class. Note that $\mathscr{D}^{\lambda}$ in a sense barely fails to satisfy any of these conditions, on account of having degrees with thin tails.

The hyperbolic Poisson-Voronoi tessellation is first considered by [13], in which Bernoulli percolation on $\mathscr{D}^{\lambda}$ is studied. There it is shown that Bernoulli percolation undergoes two phase transitions at values $p_{c}(\lambda)<p_{u}(\lambda)$ from having only finite clusters, to having infinitely many infinite clusters, to having a unique infinite cluster. Further, an upper bound is given on $p_{c}(\lambda)$ that suggests (and led the first author and Oded Schramm to conjecture) that $p_{c}(\lambda) \rightarrow \frac{1}{2}$ as $\lambda \rightarrow \infty$.

Indeed, it is the case that as $\lambda \rightarrow \infty$, all finite neighborhoods of 0 in $\mathscr{D}^{\lambda}$ will converge in law to those of the Euclidean-Poisson-Voronoi tessellation. This is because increasing $\lambda$ is tantamount to decreasing the curvature. This curvature 
effect can also be seen in how the average degree (appropriately defined) decreases as $\lambda$ increases (see Figure 1 for an illustration).

The hyperbolic Poisson-Voronoi tessellation is one of many planar stochastic triangulations with hyperbolic characteristics. The planar stochastic hyperbolic triangulation of [18] is formed by a peeling process that ensures a type of spatial Markov property and produces a stationary triangulation. There are also half-plane versions $[6,7]$ with another type of Markov property which share many of the same features, although these are not stationary.

Though the hyperbolic Poisson-Voronoi tessellation does not have a domain Markov property, the diameter of any given Voronoi cell has a subexponential tail (cf. Lemma 1.3). Hence, there is a very strong sense in which the graph is local, that is, the law of a small neighborhood of a graph depends only on the portion of $\Pi^{\lambda}$ in a small ball around that point. This leads to rapid decorrelation between a neighborhood of any vertex of $\mathscr{D}^{\lambda}$ and a neighborhood of a vertex that is far away.

Consequently, it is straightforward to show that the hyperbolic Poisson-Voronoi tessellation arises as a local limit of finite triangulations. This was observed earlier by the first author and Oded Schramm before the notion of local limit was codified (see [13], "Hyperbolic Surfaces" proof of Theorem 6.2). It is an immediate consequence of the existence of finite volume hyperbolic manifolds with arbitrarily large injectivity radius.

For approaches to finite random triangulations of the full plane that use enumerative techniques, proving the triangulation is a local limit of randomly rooted finite graphs is comparably quite difficult (although there has been recent progress in this direction [4]). However, the large scale behavior of all of these triangulation models should be similar: for example, we may speculate that after appropriately matching parameters in the two models and embedding them in a stationary way in the Poincaré disk, the distribution on exit measures on the boundary of the unit circle are mutually absolutely continuous.

Open questions. We believe that this analysis has only scratched the surface of what can be asked about the hyperbolic Poisson-Voronoi tessellation. First, the hyperbolic Poisson-Voronoi tessellation generalizes immediately to higherdimensional hyperbolic spaces, for which it should still be the case that various dual graphs have positive anchored expansion. We believe the same approach used here could be adapted to that case with the principal missing component being the analogue of Proposition 3.3.

CONJECTURE 1.8. Let $\mathbb{H}^{d}$ denote d-dimensional hyperbolic space, and let $\Pi^{\lambda}$ be a Poisson process with invariant intensity measure. The dual graph of the Voronoi tessellation of $\Pi^{\lambda}$ has positive anchored expansion.

More could be said about the speed, $s(\lambda)=\lim _{k \rightarrow \infty} d_{G}\left(X_{k}, \rho\right) / k$. Note that our result does not show that $s(\lambda)$ is deterministic, which we expect it should be. 
As increasing $\lambda$ has the effect of decreasing curvature, we expect that $s(\lambda)$ should be continuous and strictly monotone decreasing with $s\left(0^{+}\right)=1$ and $s\left(\infty^{-}\right)=0$.

By Theorem 1.2 and [16], we know that the space of bounded harmonic functions of the random walk on $\mathscr{D}^{\lambda}$ is infinite dimensional, but considerably more could be said. For example, it would be nice to know that $\mathscr{D}^{\lambda}$ has bounded harmonic functions with finite Dirichlet energy (cf. [12], Theorem 1.1) and that the wired uniform spanning forest is almost surely 1-ended (cf. [2], Theorem 7.2).

Recall that the space of bounded harmonic functions can be endowed with a measure to make it isomorphic to the Poisson boundary (see [28] or [33] for relevant background). For simple random walk on $\mathscr{D}^{\lambda}$ or $\mathscr{V}^{\lambda}$, we show that simple random walk considered as a sequence in the Poincaré disk converges almost surely to a point of $S^{1}$ in the topology of $\mathbb{C}$ (see Proposition 3.13). There are many general results about boundary convergence of random walk that are nearly applicable to $\mathscr{D}^{\lambda}$ and $\mathscr{V}^{\lambda}$. For example, [12], Theorem 1.1 , or the recent work of $[3,24]$ nearly apply to $\mathscr{D}^{\lambda}$. This leads us to believe that these results could be extended to cover graphs like $\mathscr{D}^{\lambda}$. For example in the latter work, is it possible to replace the bounded degree assumption by a stationary assumption as we have done here?

CONJECTURE 1.9. Let $(G, \rho)$ be a transient stationary random graph that is almost surely planar. Then $(G, \rho)$ embeds as a stationary subgraph of the Poincaré disk and for almost every realization of $(G, \rho)$ simple random walk almost surely converges to a point on $S^{1}$ in the topology of $\mathbb{C}$.

For bounded degree graphs, [24] has recently shown that one can embed this graph in the unit disk in such a way that random walk converges to the boundary of the disk and further shows the Poisson boundary can be represented in terms of the harmonic measure $v_{0}$ at infinity. Precisely, for Borel $A \subset S^{1}, v_{0}(A)$ is the probability that random walk converges in the Euclidean metric to a point in $A$.

In the case that the random graph is a transient, bounded degree triangulation, then [12] show that random walk almost surely converges to a point on the boundary. Subsequent work of [3] shows that the Poisson boundary can be identified with the unit circle and the harmonic measure at infinity. Since this paper appeared, [5] have shown that for essentially all unimodular, transient, one-ended circle packed random triangulations, random walk converges to a point on the boundary of the unit circle, and the exit measure is a representation of the Poisson boundary.

For comparison, the Poisson boundary of hyperbolic Brownian motion on the Poincaré disk started at 0 is naturally identified with Lebesgue measure on $S^{1}$. Given the dimension drop phenomenon observed for harmonic measure on infinite supercritical Galton-Watson trees [32], we expect something similarly singular here.

CONJECTURE 1.10. For almost every realization of $\mathscr{D}^{\lambda}, v_{0}$ is singular with respect to Lebesgue measure on $S^{1}$. 
While $\mathscr{D}^{\lambda}$ and $\mathscr{V}^{\lambda}$ are hyperbolic on a large scale, they cannot satisfy many types of uniform hyperbolic properties, such as Gromov hyperbolicity, by virtue of large sections of the triangular lattice embedding into $\mathscr{D}^{\lambda}$. (The authors expected all finite planar graphs to embed in $\mathscr{D}^{\lambda}$, but some care is needed here: not all planar triangulations can be realized as Euclidean-Delaunay graphs [20,21].) That said, there is still room to characterize which qualitative features of hyperbolic graphs are present. For example, it is natural to ask how nearly geodesics in $\mathscr{D}^{\lambda}$ match geodesics from the hyperbolic plane.

ConjectuRe 1.11. Consider connecting the Voronoi cells containing $-r$ and $r$ by a geodesic in $\mathscr{D}^{\lambda}$, and let $D_{r}$ be the graph (or hyperbolic) distance from this geodesic to the cell containing 0 ; then $\left\{D_{r}: r \in \mathbb{N}\right\}$ is tight.

In a similar spirit, for an angle $\theta>0$ is there a $\delta_{\theta}$ almost surely finite so that all geodesic triangles with one vertex at 0 and the other two vertices having angular separation $\theta$ are $\delta_{\theta}$-thin. This is a natural "anchored" analogue of Gromov hyperbolicity.

While Theorem 1.1 shows positive speed for a large class of stationary random graphs, it is natural to ask whether the volume growth condition is necessary. We wonder if it can be weakened or removed entirely. As for the conclusions, it is natural to ask whether or not the heat kernel bound (2) that holds for bounded degree graphs with positive anchored expansion extends to stationary random graphs with positive anchored expansion.

2. Speed. We begin with the proof of the criteria for positive speed, Theorem 1.1. Following Virág [36], for any $S \subseteq \mathcal{V}(G)$ finite, let $\Delta_{i}(S):=i|S|-|\partial S|$. Define an isolated $i$-core to be a finite set of vertices $S$ so that for all $A \subsetneq S$ $\Delta_{i}(S)>\Delta_{i}(A)$. As it is possible to take $A=\varnothing$ in this definition, it follows that an isolated $i$-core must have $\Delta_{i}(S)>0$.

Let IS ${ }_{i}$ denote the union of all isolated $i$-cores in $G$. The following are easily checked.

Proposition 2.1. For any locally finite, connected, simple graph $G$ :

(1) Any finite union of isolated $i$-cores is again an isolated $i$-core.

(2) If $i^{*}(G)>i>0$, then any vertex is contained in at most finitely many connected isolated $i$-cores.

(3) If $i=i^{*}(G)>0$, then $G \backslash \mathrm{IS}_{i}$ is a (nonempty) graph with edge isoperimetric constant $i$, where the volume of a finite set of vertices is still measured as the sum of degrees in $G$.

See Corollary 3.2 of [36] for a proof of the first two facts. The proof of the third is on the first displayed equation of [36], page 1594. 
Positive speed is essentially due to the fact that the induced walk on $G \backslash \mathrm{IS}_{i}$ has positive speed. This is shown by considering the behavior of the heat kernel. One then also needs to check that the walk returns to $G \backslash \mathrm{IS}_{i}$ frequently enough, and here we use stationarity in an essential way. Indeed, without stationarity and without bounded degree, it can be shown that positive anchored expansion is insufficient for positive speed.

Let $T_{1}, T_{2}, \ldots$ be the times at which $X_{k} \in G \backslash \mathrm{IS}_{i}$. We would like to show that $T_{n} / n \rightarrow c<\infty$. By the ergodic theorem, we have that

$$
\lim _{n \rightarrow \infty} \frac{1}{n+1} \sum_{j=0}^{n} \mathbf{1}\left\{X_{j} \in G \backslash \mathrm{IS}_{i}\right\}=\mathbb{P}\left[\rho \in G \backslash \mathrm{IS}_{i} \mid \mathscr{F}\right],
$$

where $\mathscr{F}$ is the invariant $\sigma$-algebra. We first show that due to stationarity, this probability cannot be 0 for $i$ sufficiently small. It will turn out that we will additionally want control of the degrees of the random walk at the times it returns to the complement of $\mathrm{IS}_{i}$, and so with foresight, we show the following.

LEMMA 2.2. Let $(G, \rho)$ be stationary. On the event $i^{*}(G)>i$, there is an $\mathscr{F}$-measurable $M<\infty$ so that

$$
\mathbb{P}\left[\rho \in G \backslash \operatorname{IS}_{i}, \operatorname{deg}_{G}(\rho) \leq M \mid \mathscr{F}\right]>0
$$

almost surely.

ProOF. Suppose first that $\mathbb{P}\left[\rho \in G \backslash \mathrm{IS}_{i} \mid \mathscr{F}\right]=0$. Then by stationarity,

$$
\mathbb{P}\left[X_{k} \in G \backslash \mathrm{IS}_{i} \mid \mathscr{F}\right]=0
$$

for all fixed $k \geq 0$. Hence, random walk stays in $\mathrm{IS}_{i}$ for all time almost surely. By Proposition 2.1 when $i^{*}(G)>i$, there are only finitely many connected isolated $i$-cores containing $\rho$. In particular, the connected component of $\operatorname{IS}_{i}$ containing $\rho$ is finite, and so when $i^{*}(G)>i$ random walk almost surely enters $G \backslash \mathrm{IS}_{i}$ in finite time, giving a contradiction.

Hence, we have shown that $\mathbb{P}\left[\rho \in G \backslash \mathrm{IS}_{i} \mid \mathscr{F}\right]>0$. By monotone convergence and the almost sure finiteness of $\operatorname{deg}_{G}(\rho)$, it follows that

$$
\lim _{M \rightarrow \infty} \mathbb{P}\left[\rho \in G \backslash \operatorname{IS}_{i}, \operatorname{deg}_{G}(\rho) \leq M \mid \mathscr{F}\right]=\mathbb{P}\left[\rho \in G \backslash \mathrm{IS}_{i} \mid \mathscr{F}\right] .
$$

Hence in particular, there must be an $M$ sufficiently large that this conditional probability is positive.

Consider the induced random walk $Y_{k}=X_{T_{k}}$ on the vertices $v$ of $G \backslash \mathrm{IS}_{i}$. Let $p_{k}(u, v)$ be the heat kernel for this random walk, that is,

$$
p_{k}(u, v)=\mathbb{P}\left[Y_{k}=v \mid \sigma(G, \rho), Y_{0}=u\right] .
$$


This operator is reversible with respect to $\pi(x)=\operatorname{deg}_{G}(x)$, observing that

$$
\operatorname{deg}_{G}(u) p(u, v)=\sum_{\gamma} \prod \frac{1}{\operatorname{deg}_{G}\left(\gamma_{i}\right)}=\operatorname{deg}_{G}(v) p(v, u)
$$

with the sum over all paths $\gamma$ connecting $u$ to $v$ via paths with interior vertices in $\mathrm{IS}_{i}$ and the product over all interior vertices in the path.

Define an inner product on functions of the vertices of $G \backslash \mathrm{IS}_{i}$ by

$$
(f, g)=\sum_{v \in G \backslash \mathrm{IS}_{i}} f(v) g(v) \operatorname{deg}_{G}(v) .
$$

Let $P$ denote the Markov operator defined by $(P f)(u)=\sum_{v} p(u, v) f(v)$. From the Cheeger inequality on $G \backslash \mathrm{IS}_{i}$ (Proposition 2.1), $P$ has operator norm strictly less than 1 on the Hilbert space with the defined inner product $(\cdot, \cdot)$ (see [34], [27], Theorem 5.1, or [33]). Hence,

$$
p_{k}(u, v)=\frac{\left(\delta_{u}, P^{k} \delta_{v}\right)}{\operatorname{deg}_{G}(u)} \leq \sqrt{\frac{\operatorname{deg}_{G}(v)}{\operatorname{deg}_{G}(u)}} q^{k},
$$

where $\delta_{x} \in L^{2}\left(\mathcal{V}\left(G \backslash \mathrm{IS}_{i}\right), \operatorname{deg}_{G}\right)$ is 1 at $x$ and 0 elsewhere, and $q=q(G, \rho)<1$. The proof of Theorem 1.1 is now a simple consequence.

Proof of Theorem 1.1. By assumption, $i^{*}(G)>0$ almost surely. Hence by Lemma 2.2 and by letting $i$ run over $\{1 / k\}_{k=1}^{\infty}$, we may restrict to considering realizations of $\left(G,\left(X_{i}\right)_{0}^{\infty}\right)$ and $i>0$ for which

$$
\mathbb{P}\left[\rho \in G \backslash \operatorname{IS}_{i}, \operatorname{deg}_{G}(\rho) \leq M \mid \mathscr{F}\right]>0 .
$$

By the subadditive ergodic theorem [31], Theorem 5.3, or [22], Theorem 6.1, the limit

$$
s=\lim _{k \rightarrow \infty} \delta\left(\rho, X_{k}\right) / k
$$

exists almost surely and is equal to some $\mathscr{F}$-measurable random variable. Hence, it suffices to show that $X_{k}$ has positive limsup $\delta$-speed almost surely.

By assumption, there are radii $r_{k}$ with $r_{k} \geq k$ and a constant $C$ so that

$$
\sup _{k \in \mathbb{N}}\left|B_{\delta}\left(\rho, r_{k}\right)\right|^{1 / r_{k}} \leq C<\infty \text {. }
$$

Hence, for any $\ell \in \mathbb{N}$,

$$
\mathbb{P}\left[\delta\left(Y_{\ell}, \rho\right) \leq r_{k}, \operatorname{deg}_{G}\left(Y_{\ell}\right) \leq M \mid \mathscr{F}\right] \leq C^{r_{k}} \sqrt{M} q^{\ell} .
$$

In particular, once $\ell$ is a sufficiently large multiple of $r_{k}$, this will be exponentially small in $r_{k}$. Thus, there is $c>0$ so that

$$
\mathbb{P}\left[\exists \ell>c r_{k}: \delta\left(Y_{\ell}, \rho\right) \leq r_{k}, \operatorname{deg}_{G}\left(Y_{\ell}\right) \leq M \mid \mathscr{F}\right] \leq 0.99^{r_{k}} \frac{\sqrt{M}}{1-q}
$$

by summing the previous bound in $\ell$. In particular, by Borel-Cantelli, we have that there is a $k_{0}$ so that for all $k>k_{0}$ and all $\ell>c r_{k}, \delta\left(Y_{\ell}, \rho\right)>r_{k}$. 
On the other hand, recalling (3), we know that

$\lim _{n \rightarrow \infty} \frac{1}{n} \sum_{j=0}^{n} \mathbf{1}\left\{X_{j} \in G \backslash \operatorname{IS}_{i}, \operatorname{deg}_{G}\left(X_{j}\right) \leq M\right\}=\mathbb{P}\left[\rho \in G \backslash \operatorname{IS}_{i}, \operatorname{deg}_{G}(\rho) \leq M \mid \mathscr{F}\right]$,

which is by assumption positive. Therefore, there must be a $D \in \mathbb{N}$ and a $k_{1}$ almost surely finite so that for all $k>k_{1}$,

$$
\sum_{j=0}^{D r_{k}} \mathbf{1}\left\{X_{j} \in G \backslash \mathrm{IS}_{i}, \operatorname{deg}_{G}\left(X_{j}\right) \leq M\right\}>c r_{k},
$$

and so that

$$
\sum_{j=D r_{k}}^{2 D r_{k}} \mathbf{1}\left\{X_{j} \in G \backslash \operatorname{IS}_{i}, \operatorname{deg}_{G}\left(X_{j}\right) \leq M\right\}>0 .
$$

Then, with $j^{*}$ a time between $D r_{k}$ and $2 D r_{k}$ at which the indicator holds true, we have $X_{j^{*}}=Y_{\ell}$ for some $\ell>c r_{k}$. Since $\operatorname{deg}_{G}\left(X_{j^{*}}\right) \leq M$, it must be that $\delta\left(Y_{\ell}, \rho\right)>$ $r_{k}$ if $k>k_{0}$. Hence, we conclude there is an infinite collection of times $j^{*}$ at which

$$
\delta\left(X_{j^{*}}, \rho\right)>r_{k}>\frac{j^{*}}{2 D} .
$$

We also show that for a stationary graph with bounded harmonic functions, there must be infinitely many.

ProOF OF THEOREM 1.2. Let $\mathcal{G}_{\bullet}$ be the set of connected locally finite rooted graphs. This can be endowed with a topology, the local topology, under which it is polish space (see [8] for elaborations). Such graphs have countably many vertices, and so we may consider always representing these graphs on a universal countably infinite vertex set $\mathcal{V}$. For a connected, locally finite $G$, let $\mathcal{B}(G) \subseteq \ell^{\infty}(\mathcal{V})$ denote the space of bounded harmonic functions on $G$. Let $S(G) \subset \mathcal{B}(G)$ be the unit ball under the $\ell^{\infty}(\mathcal{V})$ norm. We will define a measurable mapping $\varphi: \mathcal{G}_{\bullet} \rightarrow \ell^{\infty}(\mathcal{V})$ so that $\varphi(G, \rho)$ is a nonconstant harmonic function in $S(G)$.

For a rooted, unlabeled graph $(G, \rho)$, define

$$
R(G, \rho)=\inf \left\{r \in \mathbb{Z}_{\geq 0}: \sup _{h \in S} \max _{x \in B_{G}(\rho, r)}|h(x)-h(\rho)|>0\right\} .
$$

We claim that the function $R$ is a Borel measurable function of rooted connected locally finite graphs under the local topology. Specifically, we claim

$$
\sup _{h \in S} \max _{x \in B_{G}(\rho, r)}|h(x)-h(\rho)|=\limsup _{n \rightarrow \infty} \sup _{h \in S_{n}} \max _{x \in B_{G}(\rho, r)}|h(x)-h(\rho)|,
$$

where $S_{n}$ is the set of harmonic functions on the finite graph $B_{G}(\rho, n)$ with boundary values in $[-1,1]$; the right-hand side is a lim sup of continuous functions and 
is hence Borel measurable. On the one hand, given $h \in S(G)$ one can solve the Dirichlet problem on $B_{G}(v, n)$ for any $n \geq r$ with boundary values given by $h$ to get the left-hand side is less than the right. On the other hand, given a sequence of harmonic functions $h_{n} \in S_{n}$, one can extract by diagonalization a subsequence so that $h_{n_{k}}(x)$ converges at each $x \in \mathcal{V}$. This pointwise limit is necessarily harmonic and is in $S(G)$ by the maximum principle, and this leads to the reverse inequality.

If $R(G, \rho)=-\infty$, then $S(G)$ consists only of constant harmonic functions. Hence, in this case let $\varphi(G, \cdot)=1 \in \mathcal{B}(G)$. In the case that $R(G, \rho)$ is finite, we let $\varphi(G, \rho)$ be any arbitrary $h \in S(G)$ with $\max _{x \in B_{G}(\rho, R)}|h(x)-h(\rho)|>0$. This can be done, with effort, in such a way that for each $v \in \mathcal{V},(G, \rho) \mapsto h(v)$ is Borel measurable. Further, we may pick $\varphi$ to be equivariant with respect to rooted isomorphisms, that is, if there is a bijection $\psi: \mathcal{V} \rightarrow \mathcal{V}$ inducing an isomorphism of rooted graphs $\psi:\left(G, \rho_{1}\right) \rightarrow\left(G, \rho_{2}\right)$ then $\varphi\left(G, \rho_{1}\right)=\varphi\left(G, \rho_{2}\right) \circ \psi$. This in particular assures that $\varphi$ is well-defined on rooted equivalence classes of graphs. As a consequence, the sequence $\left(h_{k}\right)_{k=0}^{\infty}=\left(\varphi\left(G, X_{k}\right)\right)_{k=0}^{\infty}$ is stationary.

For every fixed $r>0$, we define the variables

$$
Y_{k}^{r}=\max _{i=1,2, \ldots, r}\left|h_{k}\left(X_{k+i}\right)-h_{k}\left(X_{k}\right)\right|
$$

which makes $\left(Y_{k}^{r}\right)_{k=0}^{\infty}$ stationary.

Suppose that $\mathcal{B}(G)$ were finite-dimensional. Then there would be a basis

$$
f_{1}, f_{2}, \ldots, f_{d}
$$

of bounded harmonic functions. As each process $\left(f_{j}\left(X_{k}\right)\right)_{k=0}^{\infty}$ is a bounded martingale, it converges almost surely. Hence, for every fixed $r>0$, it also follows that for each $1 \leq j \leq d$,

$$
\lim _{k \rightarrow \infty} \max _{i=1,2, \ldots, r}\left|f_{j}\left(X_{k+i}\right)-f_{j}\left(X_{k}\right)\right|=0
$$

almost surely. By compactness of $S(G)$, it follows that

$$
\lim _{k \rightarrow \infty} \sup _{h \in S} \max _{i=1,2, \ldots, r}\left|h\left(X_{k+i}\right)-h\left(X_{k}\right)\right|=0 .
$$

Thus, we conclude that for every fixed $r>0$,

$$
\lim _{k \rightarrow \infty} Y_{k}^{r}=0 \text {. }
$$

Then for every fixed $r>0$ and every $\varepsilon>0$, we have that

$$
\lim _{k \rightarrow \infty} \frac{1}{k+1} \sum_{i=0}^{k} \mathbf{1}\left\{Y_{k}^{r}>\varepsilon\right\}=0 .
$$

By the ergodic theorem, however, we also have that

$$
\lim _{k \rightarrow \infty} \frac{1}{k+1} \sum_{i=0}^{k} \mathbf{1}\left\{Y_{k}^{r}>\varepsilon\right\}=\mathbb{P}\left[\exists i \leq r:\left|h_{0}\left(X_{i}\right)-h_{0}(\rho)\right|>\varepsilon \mid \mathscr{F}\right] .
$$


Hence on the event that $\mathcal{B}(G)$ is finite dimensional, we conclude that $h_{0}\left(X_{i}\right)=$ $h_{0}(\rho)$ for all $i \in \mathbb{N}$ up to null events. This, however, implies that $R=-\infty$, that is, all bounded harmonic functions are constant. Hence, almost surely, either $\mathcal{B}(G)$ consists of constant functions or $\mathcal{B}(G)$ is infinite dimensional.

3. Hyperbolic Poisson-Voronoi tessellation. Let $\mathbb{H}$ denote the hyperbolic plane. For a good introduction to hyperbolic geometry and different models of the plane, see [15]. The Poincare disk model of $\mathbb{H}$ is given by the unit disk $\left\{\left(x_{1}, x_{2}\right)\right.$ : $\left.x_{1}^{2}+x_{2}^{2}<1\right\}$ in $\mathbb{R}^{2}$ together with the Riemannian metric

$$
d s_{\mathbb{H}}^{2}=4 \frac{d x_{1}^{2}+d x_{2}^{2}}{\left(1-x_{1}^{2}-x_{2}^{2}\right)^{2}} .
$$

From this, it follows that hyperbolic area measure is absolutely continuous to Lebesgue measure and has density given by

$$
d A_{\mathbb{H}}=4 \frac{d x_{1} d x_{2}}{\left(1-x_{1}^{2}-x_{2}^{2}\right)^{2}} .
$$

We will denote the hyperbolic distance between two points $x$ and $y$ by $d_{\mathbb{H}}$, the hyperbolic area of a region $R$ by $\operatorname{Vol}_{\mathbb{H}}(R)$, and the hyperbolic disk centered at $x$ and with hyperbolic radius $r$ by $B_{\mathbb{H}}(x, r)$. Also, we denote the disk with boundary points $x, y, z$ by $\mathrm{CD}_{\mathbb{H}}(x, y, z)$, and its center by $\mathrm{CC}_{\mathbb{H}}(x, y, z)$. (We also express Euclidean distances, areas and disks the same way, except we replace $\mathbb{H}$ by $\mathbb{E}$.) Observe that

$$
\operatorname{Vol}_{\mathbb{H}}\left(B_{\mathbb{H}}(x, r)\right)=2 \pi(\cosh (r)-1) \leq \pi e^{r}
$$

and that the circumference of the same ball is given by $2 \pi \sinh (r)$.

We let $\Pi^{\lambda}$ be the Poisson process on the open unit disk with intensity $\lambda \cdot d A_{\mathbb{H}}$, conditioned to contain the point 0 . We define the 1 -skeleton graphs $\mathscr{V}^{\lambda}$ and $\mathscr{D}^{\lambda}$ from the conditioned process $\Pi^{\lambda}$ as we defined these graphs in Section 1 for $\Pi^{\lambda}$ an unconditioned Poisson process. As noted in Remark 1.7, introducing this modification does not affect the anchored expansion constant; hence, it suffices to prove Lemma 1.3 and Theorem 1.4 for these modified graphs.

The main goal of this section is to prove Theorem 1.4; but first, we give the proof of Lemma 1.3.

Proof Of Lemma 1.3. Suppose that some Delaunay triangle with one vertex the origin is not contained in $\partial B_{\mathbb{H}}(0, r)$. Then the circumcircle of this triangle contains a circle with radius $r / 2$ and center on $\partial B_{\mathbb{H}}(0, r / 2)$. Since the circumcircle cannot contain any points in the Poisson process $\Pi^{\lambda}$, we deduce that there exists a disk with radius $r / 2$ and center on $\partial B_{\mathbb{H}}(0, r / 2)$ containing no points of $\Pi^{\lambda}$.

We will define a finite set $S$ of points on $\partial B_{\mathbb{H}}(0, r / 2)$ such that any disk with radius $r / 2$ and center on $\partial B_{\mathbb{H}}(0, r / 2)$ contains $B_{\mathbb{H}}(s, r / 4)$ for some $s \in S$. Let $S$ be 
a collection such that the distances between all neighboring pairs of points along the boundary of $B_{\mathbb{H}}(0, r / 2)$ is exactly $r / 4$, except possibly for one pair of neighboring points whose pairwise distance along the boundary may be less than $r / 4$. Then any disk centered at a point on $\partial B_{\mathbb{H}}(0, r / 2)$ is at most distance $r / 8$ from a point in $S$ and so contains $B_{\mathbb{H}}(s, r / 4)$ for some $s \in S$. Also, since the circumference of $B_{\mathbb{H}}(0, r / 2)$ is $2 \pi \sinh r$, we have $|S|<c^{\prime} e^{r}$ for some constant $c^{\prime}$. Hence, by a union bound, the probability that $\Pi^{\lambda} \cap B_{\mathbb{H}}(s, r / 4)$ is empty for some $s \in S$ is at most

$$
c^{\prime} e^{r} e^{-\lambda \operatorname{Vol}_{\mathbb{H}}\left(B_{\mathbb{H}}(0, r / 4)\right)}=c^{\prime} e^{r-2 \pi \lambda(\cosh (r / 4)-1)} \leq c^{\prime \prime} e^{r+2 \pi \lambda-\pi \lambda e^{r / 4}}
$$

for some constant $c^{\prime \prime}$, completing the proof.

The rest of this section is devoted to proving Theorem 1.4. Our proof of the theorem relies on one key observation about the Delaunay triangulation: the hyperbolic area of a contiguous collection of triangles that is adjacent to the origin is on the order of the number of triangles considered. The notion of "contiguous" is made precise by the following definition: we call a collection of Delaunay triangles strongly connected if its dual graph is connected, where two triangles are connected in the dual graph if they share an edge. Call them strongly connected to the origin if one of these is a triangle containing the point 0 . Then we have the following proposition.

PROPOSITION 3.1. There is a constant $c>0$ and $a k_{0}>0$ random so that for all collections of Delaunay triangles $t_{1}, t_{2}, \ldots, t_{k}$ with $k>k_{0}$ that are strongly connected to the origin and whose union $\bigcup_{i=1}^{k} t_{i}$ is simply connected,

$$
\sum_{i=1}^{k} \operatorname{Vol}_{\mathbb{H}}\left(t_{i}\right)>c k,
$$

where for any Borel $U \subset \mathbb{H}, \operatorname{Vol}_{\mathbb{H}}(U)$ denotes hyperbolic area of $U$.

The proof of Theorem 1.4 is a relatively straightforward consequence of this proposition and the following observation.

LEMMA 3.2. For any finite set $S \subset \mathbb{H}$,

$$
\operatorname{Vol}_{\mathbb{H}}\left(\operatorname{conv}_{\mathbb{H}}(S)\right) \leq 2 \pi|S|,
$$

where $\operatorname{conv}_{\mathbb{H}}(S)$ denotes the hyperbolic convex hull $S$.

ProOf. Choose a point $p$ in the interior of $\operatorname{conv}_{\mathbb{H}}(S)$. As the boundary of $\operatorname{conv}_{\mathbb{H}}(S)$ is homeomorphic to a circle, we can order the extreme points $x_{1}, x_{2}, \ldots, x_{k}$ of $S$ in a counterclockwise ordering. Further, we have that $\operatorname{conv}_{\mathbb{H}}(S)$ is the union of closed hyperbolic triangles on vertices $\left\{p, x_{1}, x_{2}\right\},\left\{p, x_{2}, x_{3}\right\}$, 
$\ldots,\left\{p, x_{k}, x_{1}\right\}$. All hyperbolic triangles have area at most $2 \pi$, and hence the bound follows.

This also holds in higher-dimensional hyperbolic spaces, with appropriately chosen constants in place of $2 \pi$; see [9], Theorem 1 .

PROOF OF THEOREM 1.4 FROM PROPOSITION 3.1. The proofs of anchored expansion for $\mathscr{D}^{\lambda}$ and $\mathscr{V}^{\lambda}$, while similar in spirit, do differ in subtle ways, so we will present each one separately.

Proof that $\mathscr{D}^{\lambda}$ has positive anchored expansion. Let $k_{0}$ and $c$ be as in Proposition 3.1. Suppose that for some $k>k_{0}$ the vertices $v_{1}, \ldots, v_{k} \in \mathscr{D}^{\lambda}$ form a connected subgraph $S$ of $\mathscr{D}^{\lambda}$, one of which is the Voronoi cell with nucleus at 0 . Consider the subcomplex $S^{\prime}$ of the Delaunay triangulation given by the union of all triangles containing vertices of $S$, and let $S^{\prime \prime}$ denote the smallest simply connected subcomplex of the Delaunay triangulation which contains $S^{\prime}$. Then every vertex of $S$ is in the interior of the union of triangles formed by $S^{\prime \prime}$.

Observe that the convex hull of the vertices of $S^{\prime}$ is the same as the convex hull of the vertices of $S^{\prime} \backslash S$, as every vertex of $S$ is in the interior of $S^{\prime}$ which is in turn in the interior of the convex hull of $S^{\prime}$. Further, the number of triangles $t$ in $S^{\prime}$ is commensurate to the sum of degrees of $S$; precisely $t \geq \frac{1}{3} \sum_{s \in S} \operatorname{deg}_{\mathscr{D}^{\lambda}}(s)=$ $\frac{1}{3} \operatorname{Vol}_{\mathscr{D}^{\lambda}}(S)$.

On the one hand, Proposition 3.1 implies that the convex hull of the vertices of $S^{\prime} \backslash S$ has area at least $c t$ for some constant $c$. On the other hand, by Lemma 3.2, the area of the convex hull of $S^{\prime} \backslash S$ is at most $4 \pi\left|S^{\prime} \backslash S\right|$. Combining these facts, we have that $\left|S^{\prime} \backslash S\right| \geq \frac{c}{4 \pi} \operatorname{Vol}_{\mathscr{D}^{\lambda}}(S)$.

Since each vertex in $\left|S^{\prime} \backslash S\right|$ is connected to $S$ by a boundary edge of $S$ in $\mathscr{D}^{\lambda}$, we obtain $|\partial S| \geq\left|S^{\prime} \backslash S\right|$, and hence

$$
|\partial S| \geq\left|S^{\prime} \backslash S\right| \geq \frac{c}{4 \pi} \operatorname{Vol}_{\mathscr{D}^{\lambda}}(S) .
$$

This proves Theorem 1.4 for $\mathscr{D}^{\lambda}$.

Proof that $\mathscr{V}^{\lambda}$ has positive anchored expansion. Suppose once more that for some $k>k_{0}$ the vertices $d_{1}, \ldots, d_{k} \in \mathscr{V}^{\lambda}$ form a connected subgraph of $\mathscr{V}^{\lambda}$, one of which is a triangle containing 0 . Let $t_{1}, \ldots, t_{k}$ denote the corresponding strongly connected Delaunay triangles in $\mathbb{H}$. If the subcomplex $S$ of the Delaunay triangulation with triangles $t_{1}, t_{2}, \ldots, t_{k}$ is not simply connected, let $S^{\prime}$ be the smallest simply connected subcomplex of the Delaunay triangulation which contains $S$. Then $\partial S \supset \partial S^{\prime}$ and $\operatorname{Vol}_{\mathscr{V} \lambda}(S) \leq \operatorname{Vol}_{\mathscr{V} \lambda}\left(S^{\prime}\right)$. Hence, $|\partial S| / \operatorname{Vol}_{\mathscr{V} \lambda}(S) \geq\left|\partial S^{\prime}\right| / \operatorname{Vol}_{\mathscr{V} \lambda}\left(S^{\prime}\right)$, and it suffices to assume that $S$ is simply connected.

Let $\mathbf{X} \subset \mathbb{H}$ denote the set of vertices of the triangles $t_{1}, \ldots, t_{k}$, and let $\mathbf{X}^{\prime} \subset \mathbf{X}$ be the set of points in $\mathbf{X}$ that are contained in the boundary of $\bigcup_{i=1}^{k} t_{i}$. Observe that $\mathbf{X}$ and $\mathbf{X}^{\prime}$ have the same convex hull, as an interior point of a set is in the interior of the convex hull of that set. 
On the one hand, Proposition 3.1 implies that the convex hull of $\mathbf{X}$ has area at most $c|\mathbf{X}|$ for some constant $c$. On the other hand, by Lemma 3.2, the convex hull of $\mathbf{X}^{\prime}$ has area at most $4 \pi\left|\mathbf{X}^{\prime}\right|$ for any set of points $\mathbf{X}^{\prime} \subset \mathbb{H}$. Combined, these two inequalities give

$$
\frac{\left|\mathbf{X}^{\prime}\right|}{|\mathbf{X}|}>\frac{c}{4 \pi} .
$$

As the boundary of the polygon $\bigcup_{i} t_{i}$ is a closed loop, there is a bijective correspondence between $\mathbf{X}^{\prime}$ and boundary edges of $\bigcup_{i} t_{i}$. Further, the boundary edges of $\bigcup_{i} t_{i}$ are in bijective correspondence with the boundary edges of $S$ in $\mathscr{V}^{\lambda}$, so $\left|\mathbf{X}^{\prime}\right|=|\partial S|$.

As the embedding of $S$ gives a planar drawing of the graph, we have that by Euler's formula, $e-k=|\mathbf{X}|-1$, where $e$ is the number of edges in the complex $S$. As every triangle contains exactly three edges and every edge is contained in at most 2 triangles, $e \geq \frac{3}{2} k$, so that $|\mathbf{X}| \geq \frac{1}{2} k=\frac{1}{2} \operatorname{Vol}_{\mathscr{V} \lambda}(S)$.

Combining everything, we have that

$$
\frac{|\partial S|}{|S|}=\frac{\left|\mathbf{X}^{\prime}\right|}{|S|} \geq \frac{\left|\mathbf{X}^{\prime}\right|}{2|\mathbf{X}|} \geq \frac{c}{8 \pi} .
$$

3.1. Proposition 3.1: Proof overview. The bulk of the work is to prove Proposition 3.1, to which we devote the remainder of Section 3. We begin by outlining the ingredients of the proof and showing how Proposition 3.1 follows.

There are two key ingredients to proving the proposition: proving that a single Delaunay triangle cannot be too small in area, and applying that bound for a single triangle to the strongly connected collections of triangles considered in the proposition. One key idea in this proof is to use that in a Delaunay triangulation in $\mathbb{H}$, the hyperbolic circumcircle of each Delaunay triangle must be finite. Regarding the probability that a single triangle is too small, we show the following.

PROPOSITION 3.3. There is an absolute constant $C>0$ so that for any $x, y \in$ $\mathbb{H}$ and $\theta>0$, the area of the points $z \in \mathbb{H}$, for which the area of the hyperbolic triangle with vertices $\{x, y, z\}$ is less than $\theta$ and for which $\mathrm{CD}_{\mathbb{H}}(x, y, z)$ exists, is less than $\frac{C \theta}{d_{\mathbb{H}}(x, y)}$.

We present the proof in Section 3.5.

To convert this bound for a single triangle to a bound for collections of triangles, we consider adding triangles to a collection one at a time. We then attempt to stochastically dominate this procedure from below by a random walk. When we add a new triangle, if the triangle contains no new vertices, then the triangle is determined by the existing collection of points, and we have no control over its area. However, if the new triangle contains a new vertex, then there is some new randomness: thinking of the area of the triangle as roughly base times height, if the 
base is determined by the previous collection of points, the height stochastically dominates a uniform random variable independent from the existing collection of points.

Hence, provided that the base lengths in this analogy are not too small, and provided we can expose triangles in such a way that at enough steps we reveal a new vertex, then the area of a triangulated region of the plane will stochastically dominate something like a sum of uniform variables. To show that the base lengths are not too small, we are again in a similar situation-we wish to expose vertices one at a time and do a stochastic comparison to a random walk of uniform variables. As both of these random walks are determined by the locations of the points, we need to assure that the way these triangulations are built ensure that we can simultaneously assure that there are enough long base lengths and enough large heights.

To address these concerns, we create a specially tailored combinatorial construction suited to the tasks we face. For any $k \geq 3$, define a triangulation scheme to be a function $f:\{3,4, \ldots, k\} \rightarrow\left(\begin{array}{c}{[k]} \\ 2\end{array}\right)$ with the properties:

(1) $f$ is injective on $\{4, \ldots, k\}$.

(2) $f(i)=\left\{f(i)_{1}, f(i)_{2}\right\} \in\left(\begin{array}{c}{[i-1]} \\ 2\end{array}\right)$.

(3) For all $j \in\{3,4, \ldots, k\}$, the edges $\{f(i)\}_{i=3}^{j}$ form a connected graph.

(4) For every $j$, the number of $i$ so that $j=\max (f(i))$ is at most 2.

A triangulation scheme encodes how to build a triangulated domain step-by-step, where at each step a new vertex $j \in\{3,4, \ldots, k\}$ is added to the complex and a new triangle is added connecting $\left\{j, f(j)_{1}, f(j)_{2}\right\}$. See Figure 2 for an illustration.

If $\mathbf{X}=\left\{x_{1}, x_{2}, \ldots, x_{k}\right\}$ is an ordered collection of points in $\mathbb{H}$, then for each $i \in\{3,4, \ldots, k\}$, the vertices $x_{i}, x_{f(i)_{1}}$ and $x_{f(i)_{2}}$ define a closed hyperbolic triangle $\Delta_{\pi_{\mathbf{X}}, f}(i)$. We will say that the pair $\left(\pi_{\mathbf{X}}, f\right)$ is Delaunay if every triangle

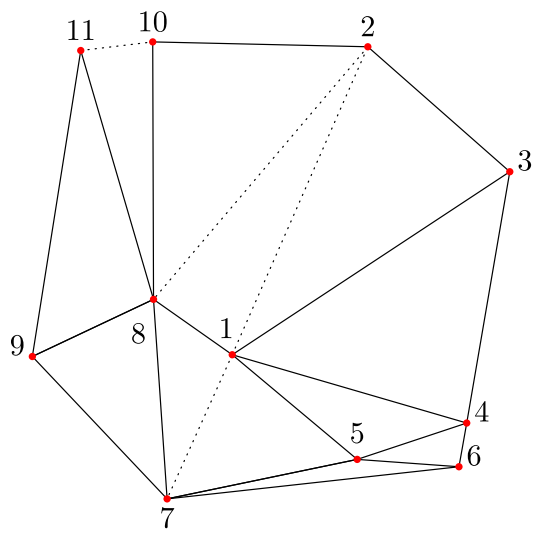

$$
\begin{aligned}
& \begin{array}{l}
\text { Triangulation } \\
\text { scheme } f:
\end{array} \\
& \hline f(3)=\{1,2\} \\
& f(4)=\{1,3\} \\
& f(5)=\{1,4\} \\
& f(6)=\{4,5\} \\
& f(7)=\{5,6\} \\
& f(8)=\{1,7\} \\
& f(9)=\{7,8\} \\
& f(10)=\{2,8\} \\
& f(11)=\{8,9\}
\end{aligned}
$$

FIG. 2. A sample triangulation scheme. The solid lines are edges that appear as $\left\{i, f(i)_{1}\right\}$ or $\left\{i, f(i)_{2}\right\}$ for some $i \in\{3,4,5, \ldots, 11\}$. The dotted lines are edges not explicitly defined by the triangulation scheme; these may or may not be used as the base of a subsequent triangle. 
$\Delta_{\pi_{\mathbf{X}}, f}(i)$ has a finite circumcircle, and planar if all the triangles $\left\{\Delta_{\pi_{\mathbf{X}}, f}(i)\right\}_{i=3}^{k}$ have pairwise disjoint interiors.

Our strategy to prove Proposition 3.1 is to show that for any finite collection of points $\mathbf{X} \subset \Pi^{\lambda}$ containing 0 , any ordering $\pi_{\mathbf{X}}$ of those points, and any triangulation scheme $f$ for which $\left(\pi_{\mathbf{X}}, f\right)$ is planar and Delaunay, we have

$$
\sum_{i=3}^{\ell}\left|\Delta_{\pi_{\mathbf{X}}, f}(i)\right|>c(\ell-2)
$$

provided $\ell=|\mathbf{X}|$ is sufficiently large.

This shows Proposition 3.1 for the special case of triangulated regions defined by triangulation schemes. To reduce the general case to this one, we show that a strongly connected collection of triangles whose union is simply connected has a vertex-spanning subcomplex whose triangles are defined by a triangulation scheme (see Figure 2 for an illustration).

LEMMA 3.4. Let $\mathcal{T}$ be a strongly connected collection of triangles from $\mathscr{D}^{\lambda}$ whose union is simply connected, and let $t \in \mathcal{T}$. Denote by $\mathbf{X}$ the set of vertices of the triangles in $\mathcal{T}$. Almost surely, there is a triangulation scheme $f$ and an ordering $\left\{x_{1}, x_{2}, \ldots, x_{k}\right\}$ of $\mathbf{X}$ such that $\Delta_{\pi_{\mathbf{X}}, f}(3)=t$ and $\left\{\Delta_{\pi_{\mathbf{X}}, f}(i)\right\}_{i=3}^{k} \subseteq \mathcal{T}$. Note that the resulting pair $\left(\pi_{\mathbf{X}}, f\right)$ is both planar and Delaunay almost surely.

This is proven is Section 3.4.

We also need to check that, despite the additional ordering we impose in our triangulation scheme description, we are still able to use a union bound over all possible triangulation schemes on all possible Delaunay vertices. Hence, we require an estimate of the number of planar Delaunay triangulation schemes on a given number of points, and so we show the following.

LEMMA 3.5. There is a constant $C>0$ so that for any (unordered) collection $\mathbf{X}$ of $k$ points in $\mathbb{H}$, the number of orderings $\pi_{\mathbf{X}}$ of $\mathbf{X}$ and triangulation schemes $f$ for which $\left(\pi_{\mathbf{X}}, f\right)$ is planar is at most $(C k)^{k}$.

We give the proof in Section 3.4. We note that without the planarity assumption, the number of such schemes would carry an extra $k^{k}$ factor, derailing any attempt at a union bound.

Finally, to complete the proof of (5), we show a stochastic domination result. Specifically, we show that for a fixed triangulation scheme, the sum of areas of its triangles stochastically dominates a certain tree-indexed product of uniform variables that we now describe. For any $i \in\{2,3, \ldots, k\}$, let $g(i)$ denote $\max (f(i))$. Define a directed graph $\mathcal{G}_{f}$ on the vertex set $\{2,3, \ldots, k\}$ with edge set given by $\{(i, g(i)): i \in\{3,4, \ldots, k\}\}$. Since $f$ is a triangulation scheme, the in-degree of any vertex is at most 2 and the out-degree of every vertex is 1 . Further, the edges 


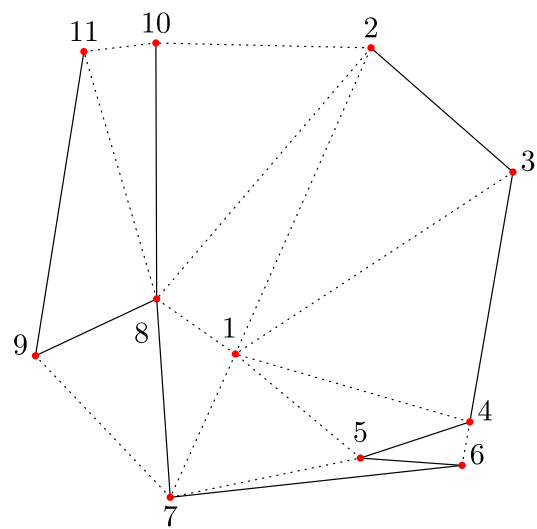

Triangulation scheme $f$ :

$f(3)=\{1,2\}$

$f(4)=\{1,3\}$

$f(5)=\{1,4\}$

$f(6)=\{4,5\}$

$f(7)=\{5,6\}$

$f(8)=\{1,7\}$

$f(9)=\{7,8\}$

$f(10)=\{2,8\}$

$f(11)=\{8,9\}$

FIG. 3. A sample triangulation scheme showing the tree $\mathcal{G}_{f}$ (see below). Edges of $\mathcal{G}_{f}$ are always oriented to go from larger vertex index to smaller. The solid lines are those that appear as $\{i, \max f(i)\}$ for some $i \in\{3,4,5, \ldots, 11\}$. The remaining edges of the complex are dotted.

are directed in such a way that from every vertex there is a directed path to 2 . Hence, the graph is connected as an undirected graph, and since it has $k-1$ vertices with $k-2$ edges, $\mathcal{G}_{f}$ is a tree. We give an example of this construction in Figure 3 .

Let $\alpha>0$ and $1 \geq \beta>0$ be fixed and let $U_{2}, U_{3}, \ldots, U_{k}$ be a collection of i.i.d. Unif[0,1] variables. Let $Z_{2}=\beta U_{2}^{\alpha / 2}$ and define inductively for $i>2$

$$
Z_{i}=\beta U_{i}^{\alpha / 2} Z_{g(i)}^{1 / \alpha}
$$

Using Proposition 3.3, we show the following comparison.

LEMMA 3.6. Let $x_{1}=0$ and let $r, s \geq 1$. Let $x_{2}$ be picked uniformly from $B_{\mathbb{H}}(0, s)$ according to hyperbolic area measure, and independently pick i.i.d. points $x_{3}, \ldots, x_{k}$ uniformly from $B_{\mathbb{H}}(0, r)$ according to hyperbolic area measure. Fix a triangulation scheme $f$, and let $\mathbf{X}=\left\{x_{1}, x_{2}, \ldots, x_{k}\right\}$. For any $\alpha>0$, there is a $\beta>0$ so that with $Z_{i}$ as in (6) and for all $r, s \geq 1$,

$$
\mathbb{P}\left[\sum_{i=3}^{k} \operatorname{Vol}_{\mathbb{H}}\left(\Delta_{\pi_{\mathbf{X}}, f}(i)\right) \leq t \text { and }\left(\pi_{\mathbf{X}}, f\right) \text { Delaunay }\right] \leq \frac{\mathbb{P}\left[\sum_{i=3}^{k} Z_{i} \leq t\right]}{\operatorname{Vol}_{\mathbb{H}}\left(B_{\mathbb{H}}(0, r)\right)^{k-2}}
$$

We give the proof in Section 3.3. We still need to show that this tree-index Markov process has the correct tail behavior, which we do by a further comparison to random walk. 
LEMMA 3.7. For any $\alpha>2, \beta>0$, and $M>0$ there is an $\varepsilon(\alpha, \beta, M)>0$ so that for all $k$,

$$
\mathbb{P}\left[\sum_{i=3}^{k} Z_{i} \leq \varepsilon(k-2)\right] \leq e^{-M(k-2)} .
$$

We give this proof in Section 3.3. This is the final ingredient in the proof of Proposition 3.1, which we give in the next section.

3.2. Proof of Proposition 3.1. Suppose that there is a constant $c>0$ and a collection of Delaunay triangles $t_{1}, t_{2}, \ldots, t_{k}$ that are strongly connected to the origin and whose union $\bigcup_{i=1}^{k} t_{i}$ is simply connected for which

$$
\sum_{i=1}^{k} \operatorname{Vol}_{\mathbb{H}}\left(t_{i}\right) \leq \frac{1}{2} c k
$$

Let $\mathbf{X}$ be the vertex set of these triangles, and let $\ell=|\mathbf{X}|$. As the complex formed by $t_{1}, t_{2}, \ldots, t_{k}$ is planar, we have from Euler's formula that $\ell \geq \frac{1}{2} k$. By Lemma 3.4, there is an ordering $\pi_{\mathbf{X}}$ of $\mathbf{X}$ and a triangulation scheme $f$ so that $\left(\pi_{\mathbf{X}}, f\right)$ is planar and Delaunay. Further, we may take the ordering so that $x_{1}=0$ and $\left\{x_{1}, x_{2}, x_{3}\right\}$ are the vertices of $t_{1}=\Delta_{\pi_{\mathbf{X}}, f}(3)$. Finally, the condition on the sum of the area of triangles implies

$$
\sum_{i=3}^{\ell} \operatorname{Vol}_{\mathbb{H}}\left(\Delta_{\pi_{\mathbf{X}}, f}(i)\right) \leq \frac{1}{2} c k \leq c \ell .
$$

This motivates the definition of the event $\mathcal{E}_{c, r, \ell}$ that there exists a triple $\left(\mathbf{X}, \pi_{\mathbf{X}}, f\right)$ where:

- $\mathbf{X} \subset \Pi^{\lambda} \cap B_{\mathbb{H}}(0, r)$ is a set containing 0 with $|\mathbf{X}|=\ell$,

- $\pi_{\mathbf{X}}$ is an ordering of $\mathbf{X}$ putting 0 first and

- $f$ is a triangulation scheme,

so that:

- the pair $\left(\pi_{\mathbf{X}}, f\right)$ is planar and Delaunay,

- $\sum_{i=3}^{\ell} \operatorname{Vol}_{\mathbb{H}}\left(\Delta_{\pi_{\mathbf{X}}, f}(i)\right) \leq c \ell$ and

- the diameter of $t_{1}$ is at most $\ell$.

To complete the proof, it suffices to show that there is a $c>0$ so that $\mathbb{P}\left[\bigcup_{r=1}^{\infty} \mathcal{E}_{c, r, \ell}\right]$ is summable in $\ell$. Then by Borel-Cantelli, there is some random $\ell_{0}<\infty$ larger than the diameter of all the Delaunay triangles incident to the origin so that for $\ell \geq \ell_{0}$,

$$
\sum_{i=3}^{\ell} \operatorname{Vol}_{\mathbb{H}}\left(\Delta_{\pi_{\mathbf{X}}, f}(i)\right) \geq c \ell
$$

from which follows Proposition 3.1. 
The following lemma therefore concludes the proof.

LEMMA 3.8. There are constants $c>0$ and $\delta$ so that for all $\ell \geq 3$,

$$
\mathbb{P}\left[\bigcup_{r=1}^{\infty} \mathcal{E}_{c, r, \ell}\right] \leq e^{-\delta \ell}
$$

Proof. Combining Lemmas 3.6 and 3.7, it follows that for any $M>0$ there is an $\varepsilon(M)>0$ so that with $\mathbf{X}=\left\{0=x_{1}, x_{2}, x_{3}, \ldots, x_{\ell}\right\}$ like in the statement of Lemma 3.6 we have for any fixed triangulation scheme $f$

$$
\begin{aligned}
& \mathbb{P}\left[\sum_{i=3}^{\ell} \operatorname{Vol}_{\mathbb{H}}\left(\Delta_{\pi_{\mathbf{X}}, f}(i)\right) \leq \varepsilon(\ell-2) \text { and }\left(\pi_{\mathbf{X}}, f\right) \text { Delaunay }\right] \\
& \quad \leq \frac{e^{-M(\ell-2)}}{\operatorname{Vol}_{\mathbb{H}}\left(B_{\mathbb{H}}(0, r)\right)^{\ell-2}} .
\end{aligned}
$$

Let $N_{r}=\left|\Pi^{\lambda} \cap B_{\mathbb{H}}(0, r)\right|$ and $N_{\ell}=\left|\Pi^{\lambda} \cap B_{\mathbb{H}}(0, \ell)\right|$. By Lemma 3.5, we have that for any set $\mathbf{X} \subset \Pi^{\lambda}$ of size $\ell$ there are at most $(C \ell)^{\ell}$ pairs $\left(\pi_{\mathbf{X}}, f\right)$ that are planar and Delaunay. Thus, we have for any $r \geq \ell$

$$
\mathbb{P}\left[\mathcal{E}_{c, r, \ell} \mid N_{r}, N_{\ell}\right] \leq\left(N_{\ell}-1\right)\left(\begin{array}{c}
N_{r}-2 \\
\ell-2
\end{array}\right)(C \ell)^{\ell} \frac{e^{-M(\ell-2)}}{\operatorname{Vol}_{\mathbb{H}}\left(B_{\mathbb{H}}(0, r)\right)^{\ell-2}}
$$

It is easily checked that for $\Pi^{\lambda}$,

$$
\lim _{r \rightarrow \infty} \mathbb{E}\left[\frac{N_{\ell} N_{r}^{\ell-2}}{\operatorname{Vol}_{\mathbb{H}}\left(B_{\mathbb{H}}(0, r)\right)^{\ell-2}}\right]=\lambda^{\ell-1} \operatorname{Vol}_{\mathbb{H}}\left(B_{\mathbb{H}}(0, \ell)\right)
$$

Hence taking expectations and limits in (9), we get

$$
\begin{aligned}
\lim _{r \rightarrow \infty} \mathbb{P}\left[\mathcal{E}_{c, r, \ell}\right] & \leq \lim _{r \rightarrow \infty} \frac{(C \ell)^{\ell} e^{-M(\ell-2)}}{(\ell-2) !} \mathbb{E}\left[\frac{N_{\ell} N_{r}^{\ell-2}}{\operatorname{Vol}_{\mathbb{H}}\left(B_{\mathbb{H}}(0, r)\right)^{\ell-2}}\right] \\
& \leq \frac{(C \ell)^{\ell} e^{-M(\ell-2)} \lambda^{\ell-1} \operatorname{Vol}_{\mathbb{H}}\left(B_{\mathbb{H}}(0, \ell)\right)}{(\ell-2) !} .
\end{aligned}
$$

By making $M$ sufficiently large, this can be made smaller than some $e^{-\delta \ell}$ for all $\ell \geq 3$. Note that since the events $\mathcal{E}_{c, r, \ell}$ are nested, the lemma follows from monotone convergence.

3.3. Probabilistic estimates: Bounding areas from a fixed Delaunay triangulation scheme. In this section, we give proofs of Lemmas 3.6 and 3.7.

PROOF OF LEMMA 3.6. For a fixed triangulation scheme $f$, define variables $Q_{f}(i)$ and $Y_{f}(i)$ for $i=2,3, \ldots, k$ by setting $Y_{f}(2)=Q_{f}(2)=d_{\mathbb{H}}\left(x_{1}, x_{2}\right)^{\alpha}$, setting

$$
Q_{f}(i)=\inf \left\{d_{\mathbb{H}}\left(x_{f(j)_{1}}, x_{f(j)_{2}}\right)^{\alpha}: j \in\{3,4, \ldots, k\}, i=\max (f(j))\right\},
$$


and setting

$$
Y_{f}(i)= \begin{cases}\operatorname{Vol}_{\mathbb{H}}\left(\Delta_{\pi_{\mathbf{X}}, f}(i)\right) \wedge Q_{f}(i) & \mathrm{CD}_{\mathbb{H}}\left(x_{i}, x_{f(i)_{1}}, x_{f(i)_{2}}\right) \text { exists } \\ \infty & \text { else. }\end{cases}
$$

As $Y_{f}(i) \leq \operatorname{Vol}_{\mathbb{H}}\left(\Delta_{\pi_{\mathbf{X}}, f}(i)\right)$ on the event that $(\mathbf{X}, f)$ is Delaunay, it suffices to show that there is a $\beta>0$ so that

$$
\mathbb{P}\left[\sum_{i=3}^{k} Y_{f}(i) \leq t\right] \leq \frac{\mathbb{P}\left[\sum_{i=3}^{k} Z_{i} \leq t\right]}{\operatorname{Vol}_{\mathbb{H}}(B(0, r))^{k-2}} .
$$

For each $i \in\{3,4, \ldots, k\}$, let $\mathscr{F}_{i}=\sigma\left(x_{1}, x_{2}, \ldots, x_{i}\right)$. Applying a union bound, we have that

$$
\begin{aligned}
\mathbb{P}\left[Y_{f}(i) \leq t \mid \mathscr{F}_{i-1}\right] \leq & \mathbb{P}\left[\operatorname{Vol}_{\mathbb{H}}\left(\Delta_{\pi_{\mathbf{X}}, f}(i)\right) \leq t\right. \text { and } \\
& \left.\mathrm{CD}_{\mathbb{H}}\left(x_{i}, x_{f(i)_{1}}, x_{f(i)_{2}}\right) \text { exists } \mid \mathscr{F}_{i-1}\right] \\
& +\sum_{\substack{j \in[k], i=g(j)}} \mathbb{P}\left[d_{\mathbb{H}}\left(x_{f(j)_{1}}, x_{f(j)_{2}}\right)^{\alpha} \leq t \mid \mathscr{F}_{i-1}\right] .
\end{aligned}
$$

To the first term in the bound, we apply Proposition 3.3. For the second term, we have that

$$
\sup _{y \in B_{\mathbb{H}}(0, r)} \mathbb{P}\left[d_{\mathbb{H}}\left(x_{i}, y\right)^{\alpha} \leq t \mid \mathscr{F}_{i-1}\right] \leq \frac{\operatorname{Vol}_{\mathbb{H}}\left(B_{\mathbb{H}}\left(0, t^{1 / \alpha}\right)\right)}{\operatorname{Vol}_{\mathbb{H}}\left(B_{\mathbb{H}}(0, r)\right)} .
$$

Further, as $f$ is a triangulation scheme, the number of $j$ for which $i=g(j)$ is at most 2. Thus for some absolute constant $C>0$, (10) becomes

$$
\begin{aligned}
\mathbb{P}\left[Y_{f}(i) \leq t \mid \mathscr{F}_{i-1}\right] \leq & \frac{C t}{d_{\mathbb{H}}\left(f(i)_{1}, f(i)_{2}\right) \operatorname{Vol}_{\mathbb{H}}\left(B_{\mathbb{H}}(0, r)\right)} \\
& +2 \frac{\operatorname{Vol}_{\mathbb{H}}\left(B_{\mathbb{H}}\left(0, t^{1 / \alpha}\right)\right)}{\operatorname{Vol}_{\mathbb{H}}\left(B_{\mathbb{H}}(0, r)\right)}
\end{aligned}
$$

For $t$ on compact sets, we have that $\operatorname{Vol}_{\mathbb{H}}\left(B_{\mathbb{H}}\left(0, t^{1 / \alpha}\right)\right) t^{-2 / \alpha}$ stays bounded. Furthermore, on the event that $Y_{f}(g(i))<\infty$, we have that $Y_{f}(g(i)) \leq \pi$. Hence,

$$
\operatorname{Vol}_{\mathbb{H}}\left(B_{\mathbb{H}}\left(0, Y_{f}(g(i))^{1 / \alpha^{2}} t^{1 / \alpha}\right)\right) t^{-2 / \alpha} \mathbf{1}\left\{Y_{f}(g(i))<\infty\right\}
$$

stays bounded for compact sets of $t$. Also on the event that $Y_{f}(g(i))<\infty$, we have that $Y_{f}(g(i))^{1 / \alpha} \leq d_{\mathbb{H}}\left(f(i)_{1}, f(i)_{2}\right)$. Therefore, for all $t \leq 1$, we have by bounding each term in (11) separately

$$
\operatorname{Vol}_{\mathbb{H}}\left(B_{\mathbb{H}}(0, r)\right) \cdot \mathbb{P}\left[Y_{f}(i) \leq t\left(Y_{f}(g(i))\right)^{1 / \alpha} \mid \mathscr{F}_{i-1}\right] \mathbf{1}\left\{Y_{f}(g(i))<\infty\right\} \leq C t^{2 / \alpha}
$$

for some absolute constant $C>0$. 
Adjusting constants, we have that there is an absolute constant $\beta_{1}>0$ so that for $i>2$,

$$
\begin{aligned}
\operatorname{Vol}_{\mathbb{H}} & \left(B_{\mathbb{H}}(0, r)\right) \cdot \mathbb{P}\left[Y_{f}(i) \leq t \beta_{1}\left(Y_{f}(g(i))\right)^{1 / \alpha} \mid \mathscr{F}_{i-1}\right] \mathbf{1}\left\{Y_{f}(g(i))<\infty\right\} \\
& \leq \mathbb{P}\left[U_{i}^{\alpha / 2} \leq t\right] .
\end{aligned}
$$

For $i=2, Y_{f}(2)=d_{\mathbb{H}}\left(x_{2}, x_{1}\right)^{\alpha}$ satisfies

$$
\mathbb{P}\left[d_{\mathbb{H}}\left(x_{2}, x_{1}\right)^{\alpha} \leq t\right] \leq \frac{\operatorname{Vol}_{\mathbb{H}}\left(B_{\mathbb{H}}\left(0, t^{1 / \alpha}\right)\right)}{\operatorname{Vol}_{\mathbb{H}}\left(B_{\mathbb{H}}(0, r)\right)} .
$$

Hence, as $r \geq 1$, we can find an appropriate constant $\beta_{2}>0$ so that

$$
\mathbb{P}\left[d_{\mathbb{H}}\left(x_{2}, x_{1}\right)^{\alpha} \leq \beta_{2} t\right] \leq t^{2 / \alpha} .
$$

Setting $\beta=\beta_{1} \wedge \beta_{2}$, we now have for all $i \geq 2$,

$$
\begin{aligned}
\operatorname{Vol}_{\mathbb{H}}\left(B_{\mathbb{H}}(0, r)\right) \cdot \mathbb{P}\left[Y_{f}(i) \leq t \beta\left(Y_{f}(g(i))\right)^{1 / \alpha} \mid \mathscr{F}_{i-1}\right] \mathbf{1}\left\{Y_{f}(g(i))<\infty\right\} \\
\quad \leq \mathbb{P}\left[U_{i}^{\alpha / 2} \leq t\right] .
\end{aligned}
$$

The result now follows by a standard induction argument.

Proof OF Lemma 3.7. Suppose that for some $\tau>0$,

$$
\sum_{i=3}^{k} \log \left(1 / Z_{i}\right)<\tau(k-2) \text {. }
$$

Then the number of $i$ so that $\log \left(1 / Z_{i}\right)>2 \tau$ is at most $\frac{1}{2}(k-2)$. Hence, for at least $\frac{1}{2}(k-2)$ many $i, Z_{i} \geq e^{-2 \tau}$ implying that

$$
\sum_{i=3}^{k} Z_{i}>e^{-2 \tau} \cdot \frac{(k-2)}{2} .
$$

Hence, it suffices to show that there is a $\tau(\alpha, \beta, M)>0$ so that

$$
\mathbb{P}\left[\sum_{i=3}^{k} \log \left(1 / Z_{i}\right) \geq \tau(k-2)\right] \leq e^{-M(k-2)},
$$

as then picking $\varepsilon=e^{-2 \tau} / 2$, the desired result follows.

Recall (6), which states that $Z_{i}=\beta U_{i}^{\alpha / 2} Z_{g(i)}^{1 / \alpha}$. Let $\mathcal{P}_{i}$ be the set of vertices in the unique path in $\mathcal{G}_{f}$ connecting $i$ to 2 , and let $d(x, y)$ be the graph distance between vertices $x$ and $y$ in $\mathcal{G}_{f}$. Then we can write

$$
Z_{i}=\prod_{j \in \mathcal{P}_{i}}\left(\beta U_{j}^{\alpha / 2}\right)^{\alpha^{-d(j, i)}},
$$


so that

$$
\begin{aligned}
\log \left(1 / Z_{i}\right) & \leq \sum_{j \in \mathcal{P}_{i}}-(\log \beta) \alpha^{-d(j, i)}+\sum_{j \in \mathcal{P}_{i}}-\left(\log U_{j}\right) \alpha^{1-d(j, i)} / 2 \\
& \leq \frac{-(\log \beta) \alpha}{\alpha-1}+\sum_{j \in \mathcal{P}_{i}}-\left(\log U_{j}\right) \alpha^{1-d(j, i)} / 2 .
\end{aligned}
$$

Therefore, we can express the sum of $\log \left(1 / Z_{i}\right)$ as

$$
\sum_{i=3}^{k} \log \left(1 / Z_{i}\right) \leq(k-3) C_{\alpha, \beta}+\sum_{j=2}^{k} f(j, \alpha) \log \left(1 / U_{j}\right),
$$

where $C_{\alpha, \beta}=\frac{-(\log \beta) \alpha}{\alpha-1}$ and $f(j, \alpha)$ is some coefficient. Since $\mathcal{G}_{f}$ has maximum in-degree 2 , we have the uniform upper bound

$$
f(j, \alpha) \leq \sum_{\ell=0}^{\infty} 2^{\ell}(\alpha / 2) \alpha^{-\ell}=\frac{\alpha / 2}{1-2 / \alpha},
$$

as the number of vertices $m$ for which there is a directed path from $m$ to $j$ of length $\ell$ is at most $2^{\ell}$.

Thus, we have that

$$
\sum_{i=3}^{k} \log \left(1 / Z_{i}\right) \leq(k-3) C_{\alpha, \beta}+D_{\alpha} \sum_{j=2}^{k} \log \left(1 / U_{j}\right)
$$

for some positive constants $C_{\alpha, \beta}$ and $D_{\alpha}$. Recall that $\log \left(1 / U_{j}\right)$ is distributed as an $\operatorname{Exp}(1)$ variable, which has some finite exponential moments. Thus, it follows that there is $\tau^{\prime}(M)>0$ so that for all $M>0$ and all $k \geq 2$,

$$
\mathbb{P}\left[\sum_{j=2}^{k} \log \left(1 / U_{j}\right)>\tau^{\prime}(M)(k-1)\right] \leq e^{-M(k-1)}
$$

and, therefore, it is possible to choose $\tau$ appropriately.

3.4. Combinatorial geometry elements. This section contains the proofs of Lemma 3.4 and Lemma 3.5. We split the proof of Lemma 3.4 into two parts, the first of which is the following lemma.

LEMMA 3.9. Let $\mathcal{T}$ be a strongly connected collection of $\ell$ triangles from $\mathscr{V}^{\lambda}$ whose union is simply connected in $\mathbb{H}$. Also, let $t_{*}$ be any triangle in $\mathcal{T}$, and let $V$ be any subcollection of the set of boundary edges of the triangulated polygon formed by the triangles in $\mathcal{T}$. Then there is an ordering $\left\{t_{1}, t_{2}, \ldots, t_{\ell}\right\}$ of the triangles in $\mathcal{T}$ with $t_{1}=t_{*}$ so that the following two properties are satisfied: 
(1) For each $1 \leq i \leq \ell$, the triangles $\left\{t_{1}, t_{2}, \ldots, t_{i}\right\}$ are strongly connected, and their union is simply connected in $\mathbb{H}$.

(2) Let $J$ be the set of all $1<i \leq \ell$ for which $t_{i}$ shares exactly one edge with $t_{1} \cup t_{2} \cup \cdots \cup t_{i-1}$. Denote this shared edge by $e_{i}$. Let

$$
\mathcal{S}=\left\{e_{i}: i \in J\right\} .
$$

Then $\mathcal{S} \cup V$ is connected.

PROOF. We proceed by induction on $\ell$. For the base case, note that when $\ell=1$, the result is satisfied since $t_{1}$ is connected and all 6 subcollections of its edges are connected.

Suppose that $\mathcal{T}$ is a strongly connected collection of triangles with $|\mathcal{T}| \geq 2$ from $\mathscr{V}^{\lambda}$ and $V$ is any collection of boundary edges. Let $P$ be the hyperbolic polygon given by the union of triangles in $\mathcal{T}$. Suppose there is a triangle $t \in \mathcal{T}$ of which two of its sides are boundary edges of $P$. Assume that $t_{1} \neq t$; an analogous argument covers the case that $t_{*}=t$. Let $\mathcal{T}^{\prime}=\mathcal{T} \backslash\{t\}$. As $t$ only shares one edge with the rest of $\mathcal{T}$, it must be a leaf in any spanning tree in the dual graph on $\mathcal{T}$. Hence, $\mathcal{T}^{\prime}$ is strongly connected. Moreover, since $t$ intersects the boundary of $P$, the triangles in $\mathcal{T}^{\prime}$ also form a simply connected region in $\mathbb{H}$. Let $e$ be the interior edge of $t$, and define $V^{\prime}$ as the union of the edges of $V$ that are edges of some triangle in $\mathcal{T}^{\prime}$ with $\{e\}$. Apply the induction hypothesis to $\left(\mathcal{T}^{\prime}, V^{\prime}\right)$ to order the triangles in $\mathcal{T}^{\prime}$ so that the properties listed in the lemma are satisfied. Extend this ordering to $\mathcal{T}$ by setting $t_{\ell}=t$. It is easy to see that the ordering of $\mathcal{T}$ just defined satisfies the properties listed in the lemma.

Next, suppose that every triangle with an edge in the boundary of $P$ has exactly one such edge. Let $\gamma$ be any closed loop in the boundary of the polygon $P$, so that $\gamma$ is a union of boundary edges. Let $e$ be a boundary edge that is not an isolated edge in $\gamma \cap V$; there will always be at least 2 such edges. Thus, we may choose the edge $e$ which is contained in a boundary triangle $t \neq t_{*}$. Let $\mathcal{T}^{\prime}=\mathcal{T} \backslash\{t\}$.

There are two cases to consider:

- Suppose first that $\mathcal{T}^{\prime}$ is strongly connected. Then set $V^{\prime}=V \backslash\{e\}$ and apply the induction hypothesis. As before, we extend the ordering of $\mathcal{T}^{\prime}$ to one of $\mathcal{T}$ by setting $t_{\ell}=t$. Property (1) of the lemma easily holds, so we just need to check that $\mathcal{T}$ satisfies property (2). The set $\mathcal{S}$ defined in the lemma is the same for $\mathcal{T}$ and $\mathcal{T}^{\prime}$; hence, $\mathcal{S} \cup V^{\prime}$ is connected. Since $e$ is not an isolated edge in $\gamma \cap V$, we have that $\mathcal{S} \cup V$ is connected as well.

- Now, consider the case in which $\mathcal{T}^{\prime}$ is not strongly connected. In this case, $\mathcal{T}$ can be decomposed as $\mathcal{T}_{1} \cup \mathcal{T}_{2}^{\prime} \cup\{t\}$ so that $\mathcal{T}_{1}$ and $\mathcal{T}_{2}^{\prime}$ are both strongly connected collections of triangles, but the two collections are disjoint in $\mathscr{V}^{\lambda}$. Without loss of generality, let $\mathcal{T}_{1}$ be the component containing $t_{*}$. Define $\mathcal{T}_{2}=\mathcal{T}_{2}^{\prime} \cup\{t\}$. Let $g$ be the edge shared by $t$ and $\mathcal{T}_{1}$, and $h$ the edge shared by $t$ and $\mathcal{T}_{2}^{\prime}$. Finally, set $V_{1}=\left(V \cap \mathcal{T}_{1}\right) \cup\{g\}$ and $V_{2}=\left(V \cap \mathcal{T}_{2}\right) \cup\{g\}$. 
Apply the induction hypothesis to $\left(\mathcal{T}_{1}, V_{1}\right)$, setting $t_{1}=t_{*}$. Also apply the induction hypothesis to $\left(\mathcal{T}_{2}, V_{2}\right)$, setting $t_{1}=t$. Now, define an ordering of all the triangles in $\mathcal{T}$ by concatenating the ordered lists of triangles in $\mathcal{T}_{1}$ and $\mathcal{T}_{2}$, with the triangles in $\mathcal{T}_{1}$ coming first. It is elementary to check that the resulting ordering satisfies property (1) of the lemma. We turn to showing that $\mathcal{S} \cup V$ is connected; to prove this, it suffices to check that $g \in \mathcal{S}$. Neither $e$ nor $h$ are edges in $\mathcal{T}_{1}$. Hence, when the triangle $t$ appears in the ordering, the edge $g$ is added to $\mathcal{S}$.

This completes the induction.

Proof of Lemma 3.4. Apply Lemma 3.9 with $V=\varnothing$ to obtain an ordering $\mathcal{T}=\left\{t_{1}, t_{2}, \ldots, t_{\ell}\right\}$ of the triangles of $\mathcal{T}$ with $t_{1}=t$, so that the ordering satisfies the properties listed in the lemma. Order the elements of $J$ as $i_{1}<\cdots<i_{r}$, and define the mapping from $\mathcal{S}$ to $\mathbf{X}$ that sends each $e_{i}$ to the unique vertex of the triangle $t_{i}$ not contained in $e_{i}$. This mapping is easily seen to be injective from the definition of $\mathcal{S}$. Furthermore, every vertex except those in $t_{1}$ is in the range of this mapping. Hence, $k=r+3=|\mathcal{S}|+3$.

Assign an ordering $\mathbf{X}=\left\{x_{1}, x_{2}, \ldots, x_{k}\right\}$ to the vertices of the triangles in $\mathcal{T}$ so that $t_{1}=\left\{x_{1}, x_{2}, x_{3}\right\}$ and, for $3<j \leq k$, the point $x_{j}$ is the unique vertex of triangle $t_{i_{j-3}}$ not contained in $e_{i_{j-3}}$. Then, setting $f(3)=\{1,2\}$ and $f(j)=e_{i_{j-3}}$ for $j>3$, we have both $\Delta_{\pi_{\mathbf{X}}, f}(3)=t_{1}$ and $\left\{\Delta_{\pi_{\mathbf{X}}, f}(i)\right\}_{i=3}^{k} \subseteq \mathcal{T}$. It remains to prove that $f$ is a triangulation scheme. Conditions (1) and (2) for a triangulation scheme are easily satisfied, and condition (3) follows immediately from Lemma 3.9. So we just need to check that $f$ satisfies condition (4).

Suppose for contradiction that $f$ does not satisfy condition (4). Then, for some $j$, we can find $p_{1}<p_{2}<p_{3}$ in $[k]$ with $\max \left(f\left(p_{n}\right)\right)=j$ for $n=1,2,3$. Set $q_{n}=\min \left(f\left(p_{n}\right)\right)$. Since $t_{1} \cup \cup_{2 \leq m<i_{j-3}} t_{m}$ is path-connected, we can find paths $\gamma_{1}, \gamma_{2}, \gamma_{3}$ in $t_{1} \cup \cup_{2 \leq m<i_{j-3}} t_{m}$ with endpoints $\left\{q_{2}, q_{3}\right\},\left\{q_{1}, q_{3}\right\}$ and $\left\{q_{1}, q_{2}\right\}$, respectively. Let $\gamma_{1}^{\prime}$ be the closed loop in $t_{1} \cup \cup_{2 \leq m<i_{p_{3}-3}} t_{m}$ obtained from $\gamma_{1}$ by adjoining to $\gamma_{1}$ the edges connecting $x_{j}$ to $x_{q_{2}}$ and $x_{q_{3}}$. Define $\gamma_{2}^{\prime}$ and $\gamma_{3}^{\prime}$ analogously. Then, at least one of the triangles $t_{i_{n-3}}$ must be contained in one of the three regions bounded by the closed curves $\gamma_{n}^{\prime}$. But this means that $t_{1} \cup \cup_{2 \leq m \leq i_{j-3}} t_{m}$ is not simply connected, contradicting the result of Lemma 3.9.

We now turn to proving an estimate on the number of triangulation schemes.

PROOF OF LEMMA 3.5. We begin by introducing some terminology. A planar drawing of a graph is a representation of the graph in the plane in which the vertices of the graph are mapped to distinct points in the plane, and edges of the graph are mapped to continuous paths connecting the corresponding pairs of vertices. A planar drawing is called crossing-free if there are no crossings between the paths in the plane representing the edges in the graph. By [1], Theorem 2, there is a 
$C_{1}>0$ so that the number of crossing-free subgraphs of any planar drawing of a graph on $k$ vertices is at most $C_{1}^{k}$.

The complete graph with vertices $\mathbf{X}$ has a natural planar drawing given by connecting each pair of points by its hyperbolic geodesic. Every planar $\left(\pi_{\mathbf{X}}, f\right)$ gives rise to an ordered tuple of triangles $\mathscr{S}_{\left(\pi_{\mathbf{X}}, f\right)}=\left(\Delta_{\pi_{\mathbf{X}}, f}(3), \Delta_{\pi_{\mathbf{X}}, f}(4), \ldots\right.$, $\left.\Delta_{\pi_{\mathbf{X}}, f}(k)\right)$. Note that the mapping $\left(\pi_{\mathbf{X}}, f\right) \mapsto \mathscr{S}_{\left(\pi_{\mathbf{X}}, f\right)}$ is 6-to-1. (We may freely choose the ordering of the first three vertices of $\mathbf{X}$, but the rest is determined.)

Furthermore, the union of triangles in $\mathscr{S}_{\left(\pi_{\mathbf{X}}, f\right)}$ is a crossing-free subgraph of the natural planar drawing of the complete graph on $\mathbf{X}$. By the theorem cited above, there are at most $C_{1}^{k}$ such subgraphs. Now, it is possible that, for different planar pairs $\left(\pi_{\mathbf{X}}^{\prime}, f^{\prime}\right)$ and $\left(\pi_{\mathbf{X}}^{\prime \prime}, f^{\prime \prime}\right)$, the unions of $\mathscr{S}_{\left(\pi_{\mathbf{X}}^{\prime}, f^{\prime}\right)}$ and $\mathscr{S}_{\left(\pi_{\mathbf{X}}^{\prime \prime}, f^{\prime \prime}\right)}$ give the same crossing-free subgraph. We claim that there is a constant $C_{2}>0$ so that the number of planar pairs $\left(\pi_{\mathbf{X}}, f\right)$ that yield the same crossing-free subgraph is at most $C_{2}^{k} k$ !. This will complete the proof, since then $6\left(C_{1} C_{2}\right)^{k} k^{k}$ is an upper bound for the number of orderings $\pi_{\mathbf{X}}$ and triangulation schemes $f$ for which $\left(\pi_{\mathbf{X}}, f\right)$ is planar.

Any crossing-free subgraph produced by a planar pair $\left(\pi_{\mathbf{X}}, f\right)$ is a planar drawing of contiguous hyperbolic polygons, not necessarily all triangles. By Euler's formula, the number $t$ of triangles that appear in the subgraph is at most $1+e-k$, where $e$ is the number of edges in the subgraph. As each edge came from a triangle in $\mathscr{S}_{\left(\pi_{\mathbf{X}}, f\right)}$, we must have $e \leq 3 k$; so the total number of triangles is at most $2 k+1$. Thus, the number of ways of picking an ordered $k$-element collection of triangles from this graph is at most $\left(\begin{array}{c}2 k+1 \\ k\end{array}\right) k ! \leq 4^{k} k !$.

3.5. Core geometric estimate: The proof of Proposition 3.3. In this section, we will heavily rely on the identification of $\mathbb{H}$ with the open unit disk $D$ in $\mathbb{C}$ via the Poincaré disk model. By applying an isometry, we can assume without loss of generality that $y=0$ and that $x$ lies on the positive real axis. Let $S$ denote the set of points $z \in \mathbb{H}$ such that $\mathrm{CD}_{\mathbb{H}}(0, x, z)$ exists, and let $A$ be the set of $z \in \mathbb{H}$ for which $\operatorname{Vol}_{\mathbb{H}}(\Delta(0, x, z)) \leq \theta$. Proposition 3.3 states that the hyperbolic area of $S \cap A$ is at most $\frac{C \theta}{x}$ for some absolute constant $C$.

Having identified $\mathbb{H}$ with the disk $D$, we can describe the regions $S$ and $A$ explicitly. First, we describe the region $S$. Let $G$ denote the hyperbolic geodesic ray from $x / 2$ normal to the real axis and contained in the upper half-plane. Let $q$ denote the limit point of $G$ on $\partial D$. Then the horocycle $H$ through $0, x$, and $q$ is precisely the limit of the circles through $0, x$ and $p$ as $p$ approaches $q$ along the geodesic $G$. Moreover, the set of circumcenters $\mathrm{CC}_{\mathbb{H}}(x, y, z)$ for $z \in S$ is exactly $G$. Finally, let $F$ be the circle with diameter given by the segment from 0 to $x$ in terms of which we can concisely describe $S$ as the closure of $H \backslash F$.

The region $A$ is characterized explicitly by the following lemma, a special case of Theorem 7 of [29].

LEMMA 3.10. For each $\alpha>0$, the locus of points $y \in D$ in the upper halfplane with $\operatorname{Vol}_{\mathbb{H}}(\Delta(0 x y))=\alpha$ is given by the intersection of $D$ with the ray $\ell$ from $1 / x$ that makes the angle $\alpha / 2$ with the negative real axis. 
Having described the regions $S$ and $A$ explicitly, we are now ready to prove the proposition. We divide our proof into two parts: one for $x$ less than or equal to some $\delta \in(0,1)$, and one for $x>\delta$.

In the case that $x \leq \delta$, we will use estimates comparing hyperbolic and Euclidean volume. Toward this end, we need the following elementary lemma.

LEMma 3.11. Fix $0<\delta<1$. For all $0 \leq x \leq \delta$ and $c<\frac{\sqrt{1-\delta^{2}}}{2}$, we have the following:

(1) If $z \in H$ with $\Re z>0$ and $\Im z \leq c$, then $\Re z \leq \frac{\delta}{2}+\sqrt{c-c^{2}}$.

(2) If $z \in H$ with $\Re z<0$ and $\Im z \leq c$, then $\Re z \geq-\sqrt{c-c^{2}}$.

ProOF. Write $H_{x}$ for the circumcircle of $0, x$ and $q$, that is, the horocycle $H$. Consider the region of $z \in \bigcup_{x^{\prime} \in(0, \delta)} H_{x^{\prime}}$ for which $\Im z<c$. The point in this region with maximal real part lies on $H_{\delta}$; the point in this region with minimal real part lies on $H_{\delta}$. Our restriction on $C$ implies that both these extremal points have imaginary part exactly $c$. The result then follows from two applications of the Pythagorean theorem.

Observe that $\ell$ intersects the vertical line $\Re(z)=-1$ at the point $-1+$ $\left(1+\frac{1}{x}\right) \tan (\theta / 2) i$. For all $x<1$ and all $\theta<\frac{\pi}{2}$, we have

$$
\left(1+\frac{1}{x}\right) \tan (\theta / 2) \leq \frac{2 \theta}{x}
$$

Thus, all points in $S \cap A$ have imaginary part at most $\frac{2 \theta}{x}$. It follows from Lemma 3.11 that we can choose $0<\delta$ and $c<1$ sufficiently small that for $x \leq \delta$ and $\frac{\theta}{x}<c$ the region $S \cap A$ is contained in the disk centered at the origin with Euclidean radius $\frac{\sqrt{2}}{2}$. In this case,

$$
\begin{aligned}
\operatorname{Vol}_{\mathbb{H}}(S \cap A) & =\int_{S \cap A} \frac{4 r}{\left(1-r^{2}\right)^{2}} d r d \theta \\
& \leq 16 \int_{S \cap A} r d r d \theta \\
& =16 \operatorname{Vol}_{\mathbb{E}}(S \cap A) .
\end{aligned}
$$

Since $S \cap A$ is contained in the rectangle with vertices \pm 1 and $\pm 1+i \frac{2 \theta}{x}$, its Euclidean area is bounded above by $\frac{4 \theta}{x}$.

We deduce that Proposition 3.3 holds (for some choice of the constant $C$ ) whenever both $x<\delta$ and $\frac{\theta}{x}<c$. In fact, we can discard the latter condition by stipulating that $C>c$.

Thus, we have reduced to the case $x>\delta$, where $\delta$ is the value chosen above. As in the previous case, it suffices to prove the proposition for $\frac{\theta}{x}<c$ for any choice 


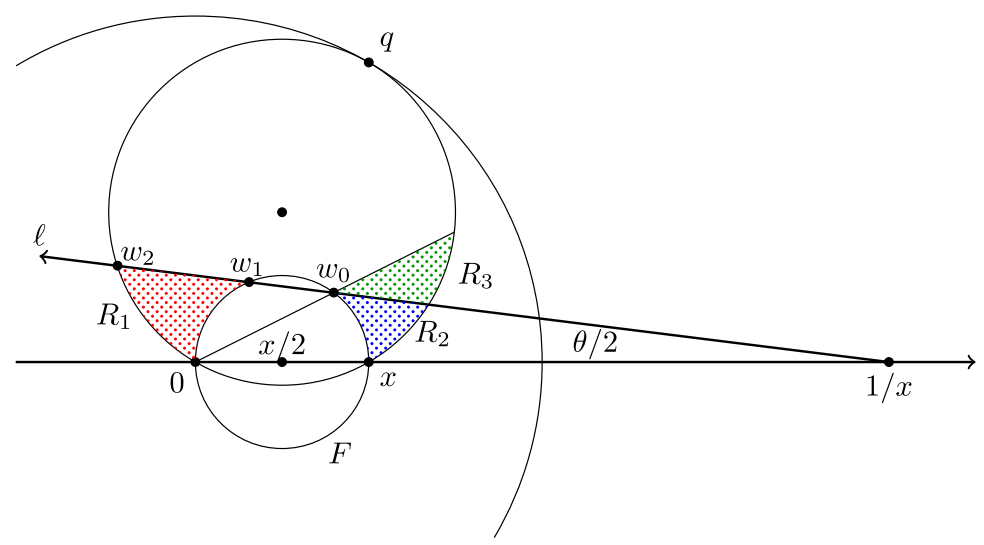

FIG. 4. The intersection of $A$ and $S$ in the plane, less the ball with diameter $[0, x]$. This is $R_{1} \cup R_{2}$. We estimate this region from above by including $R_{1} \cup R_{2} \cup R_{3}$.

of constant $c>0$. Observe that, for $x>\delta$, the condition $\frac{\theta}{x}<c$ holds as long as $\theta<c \delta$. Hence, it is enough to prove the following claim.

Claim 3.12. Fix $\delta \in(0,1)$, and suppose $x>\delta$. Then we can choose a constant $\theta_{\delta}$ and $C>0$ so that, for all $\theta<\theta_{\delta}$, the $\operatorname{Vol}_{\mathbb{H}}(S \cap A)<C \theta$.

We begin our proof of this claim with a diagram, Figure 4 . As in the figure, we can assume the ray $\ell$ intersects $F$ at two distinct points by choosing $\theta_{\delta}$ sufficiently small. Label these points as $w_{0}$ and $w_{1}$ where $w_{0}$ is the first intersection of $\ell$ with $F$. Also let $w_{2}$ be the second intersection of $\ell$ with $H$. Define $R_{1}$ to be the set of $z \in S \cap A$ with $\Re z \leq \Re w_{1}$, define $R_{2}$ to be the set of $z \in S \cap A$ with $\Re z \geq \Re w_{0}$ and define $R_{3}$ as the set of $z \in S \backslash R_{2}$ below the Euclidean line 0 and $w_{0}$.

Our strategy is to bound the hyperbolic area of $S \cap A=R_{1} \cup R_{2}$ from above by the sum of the hyperbolic area of $R_{1}$ and the hyperbolic area of $R_{2} \cup R_{3}$. First, however, we derive a bound on $\varphi_{*}:=\left|\angle x 0 w_{0}\right|$ in terms of $\theta$ and $x$. By the law of sines on the Euclidean triangle with vertices $w_{0}, x / 2$ and $1 / x$,

$$
\frac{\sin \left(\theta / 2+2 \varphi_{*}\right)}{\frac{1}{x}-\frac{x}{2}}=\frac{\sin \theta}{\frac{x}{2}}
$$

and, therefore,

$$
x^{2} \sin \left(\theta / 2+2 \varphi_{*}\right)=\sin (\theta / 2)\left(2-x^{2}\right) .
$$

Applying the sine addition formula to the first term, we can arrange terms to obtain the following quadratic in $\sin \left(2 \varphi_{*}\right)$ :

$$
x^{4} \sin ^{2}(\theta / 2)\left(1-\sin ^{2}\left(2 \varphi_{*}\right)\right)=\left(x^{2} \cos (\theta / 2) \sin (\theta / 2)-\sin (\theta / 2)\left(2-x^{2}\right)\right)^{2} .
$$


Applying the quadratic formula and taking the smaller solution, since the larger solution corresponds to $\left|\angle x 0 w_{1}\right|$, we get

$$
\sin \left(2 \varphi_{*}\right)=\sin (\theta / 2)\left(z \cos (\theta / 2)-\sqrt{1-z^{2} \sin ^{2}(\theta / 2)}\right),
$$

where $z=\frac{2-x^{2}}{x^{2}}$. Now,

$$
\begin{aligned}
\sin \left(2 \varphi_{*}\right) & =\sin (\theta / 2)\left(z \cos (\theta / 2)-\sqrt{1-z^{2} \sin ^{2}(\theta / 2)}\right) \\
& \leq \sin (\theta / 2)\left(z \cos (\theta / 2)-1+z^{2} \sin ^{2}(\theta / 2)\right) \\
& =\sin (\theta / 2) \cos (\theta / 2)(z-1)+\sin (\theta / 2)(\cos (\theta / 2)-1)+z^{2} \sin ^{3}(\theta / 2) \\
& \leq \sin (\theta / 2) \cos (\theta / 2)(z-1)+\sin (\theta / 2)\left(-\sin ^{2}(\theta / 2)\right)+z^{2} \sin ^{3}(\theta / 2) \\
& =\frac{1}{2} \sin \theta(z-1)+\sin ^{3}(\theta / 2)\left(z^{2}-1\right) \\
& \leq C_{\delta}^{\prime} \theta(1-x) \quad \text { for } x>\delta .
\end{aligned}
$$

It follows that, for $x>\delta$,

$$
\sin \left(2 \varphi_{*}\right) \leq C_{\delta}^{\prime} \theta(1-x)
$$

for some constant $C_{\delta}^{\prime}$ depending on $\delta$. Hence, we can choose $\theta_{\delta}$ sufficiently small so that

$$
\sin \left(2 \varphi_{*}\right) \leq \frac{2}{3}(1-x)
$$

and

$$
\varphi_{*} \leq C_{\delta}^{\prime} \theta(1-x)
$$

The region $R_{2} \cup R_{3}$. For each $0 \leq \varphi \leq \varphi_{*}$, consider the line through the origin that makes the angle $\varphi$ with the positive real axis. Let $p_{1}(\varphi)$ and $p_{2}(\varphi)$ be the points at which this line intersects the circle $F$ and the horocycle $H$, respectively. Set $\ell_{1}(\varphi)=\left|p_{1}(\varphi)\right|$ and $\ell_{2}(\varphi)=\left|p_{2}(\varphi)\right|$. Then the hyperbolic area of $R_{2} \cup R_{3}$ is given by

$$
\begin{aligned}
\operatorname{Vol}_{\mathbb{H}}\left(R_{2} \cup R_{3}\right) & =\int_{0}^{\varphi_{*}}\left(\frac{2}{1-\left(\ell_{2}(\varphi)\right)^{2}}-\frac{2}{1-\left(\ell_{1}(\varphi)\right)^{2}}\right) d \varphi \\
& =\int_{0}^{\varphi_{*}} \frac{2\left(\left(\ell_{2}(\varphi)\right)^{2}-\left(\ell_{1}(\varphi)\right)^{2}\right)}{\left(1-\left(\ell_{1}(\varphi)\right)^{2}\right)\left(1-\left(\ell_{2}(\varphi)\right)^{2}\right)} d \varphi \\
& =\int_{0}^{\varphi_{*}} \frac{2\left(\ell_{2}(\varphi)-\ell_{1}(\varphi)\right)}{\left(1-\ell_{1}(\varphi)\right)\left(1-\ell_{2}(\varphi)\right)} \frac{\ell_{2}(\varphi)+\ell_{1}(\varphi)}{\left(1+\ell_{1}(\varphi)\right)\left(1+\ell_{2}(\varphi)\right)} d \varphi \\
& =\int_{0}^{\varphi_{*}} \frac{4\left(\ell_{2}(\varphi)-\ell_{1}(\varphi)\right)}{\left(1-\ell_{1}(\varphi)\right)\left(1-\ell_{2}(\varphi)\right)} d \varphi
\end{aligned}
$$


The following explicit formulas for $\ell_{1}$ and $\ell_{2}$ are easily verified:

$$
\begin{aligned}
& \ell_{1}(\varphi)=x \cos \varphi, \\
& \ell_{2}(\varphi)=x \cos \varphi+\sqrt{1-x^{2}} \sin \varphi .
\end{aligned}
$$

Hence,

$$
\operatorname{Vol}_{\mathbb{H}}\left(R_{2} \cup R_{3}\right)=4 \int_{0}^{\varphi_{*}} \frac{\sqrt{1-x^{2}} \sin \varphi d \varphi}{(1-x \cos \varphi)\left(1-x \cos \varphi-\sqrt{1-x^{2}} \sin \varphi\right)} .
$$

Substituting $u=\cos \varphi$, we can rewrite this integral as

$$
\operatorname{Vol}_{\mathbb{H}}\left(R_{2} \cup R_{3}\right)=4 \int_{\cos \varphi_{*}}^{1} \frac{\sqrt{1-x^{2}} d u}{(1-x u)\left(1-x u-\sqrt{1-x^{2}} \sqrt{1-u^{2}}\right)} .
$$

Rationalizing the denominator, we get

$$
\operatorname{Vol}_{\mathbb{H}}\left(R_{2} \cup R_{3}\right)=4 \int_{\cos \varphi_{*}}^{1} \frac{\sqrt{1-x^{2}}\left(1-x u+\sqrt{1-x^{2}} \sqrt{1-u^{2}}\right) d u}{(1-x u)(x-u)^{2}} .
$$

Since $\sqrt{1-x^{2}} \sqrt{1-u^{2}} \leq 1-x u$, we get that

$$
\begin{aligned}
\operatorname{Vol}_{\mathbb{H}}\left(R_{2} \cup R_{3}\right) & \leq \sqrt{1-x^{2}} \int_{\cos \varphi_{*}}^{1} \frac{d u}{(x-u)^{2}} \\
& =8 \sqrt{1-x^{2}}\left[\frac{-1}{1-x}+\frac{1}{\cos \varphi_{*}-x}\right] \\
& =8 \sqrt{1-x^{2}} \frac{1-\cos \varphi_{*}}{(1-x)\left(\cos \varphi_{*}-x\right)}
\end{aligned}
$$

By (13) above, we have

$$
1-\cos \varphi_{*} \leq \sin \left(2 \varphi_{*}\right) \leq \frac{2}{3}(1-x)
$$

and, therefore,

$$
\left(\cos \varphi_{*}-x\right)=\left(\cos \varphi_{*}-1\right)+(1-x) \geq \frac{1}{3}(1-x) .
$$

Hence, (15) is at most

$$
\begin{aligned}
\frac{24\left(1-\cos \varphi_{*}\right)}{(1-x)^{3 / 2}} & \leq \frac{12 \varphi_{*}}{(1-x)^{3 / 2}} \\
& \leq 12 C_{\delta}^{\prime 2} \theta^{2}\left(1-x^{2}\right)^{1 / 2} \\
& \leq C_{\delta}^{\prime \prime} \theta^{2}
\end{aligned}
$$

for some constant $C_{\delta}^{\prime \prime}$ depending on $\delta$. We conclude that

$$
\operatorname{Vol}_{\mathbb{H}}\left(R_{2} \cup R_{3}\right) \leq C_{\delta}^{\prime \prime} \theta^{2}
$$


The region $R_{1}$. Next, we consider the region $R_{1}$. We claim that, for $\theta_{\delta}$ sufficiently small and $x>\delta$, the points $w_{1}$ and $w_{2}$ are contained in a ball of radius $1 / \sqrt{2}$.

First, if we let $t$ denote the unique point in the upper half-plane such that the line through $1 / x$ and $t$ is tangent to $C$ at $t$, then

$$
d_{\mathbb{E}}\left(0, w_{1}\right) \leq d_{\mathbb{E}}(0, t)=\frac{x}{\sqrt{2-x^{2}}}
$$

Since the latter is monotonic and tends to $1 / \sqrt{2}$ as $x \rightarrow 1$, we deduce that $w_{1}$ lies in the disk centered at the origin with radius $1 / \sqrt{2}$.

Next, we can bound $d_{\mathbb{E}}\left(0, w_{2}\right)$ from above by the length of the arc of the horocycle $H$ between 0 and $w_{2}$. The latter is just $\pi \alpha$, where $\alpha$ is the angle of the arc. By (13),

$$
2 \theta \geq \alpha-\varphi_{*} \geq \alpha-C_{\delta}^{\prime}(1-\delta) \theta .
$$

Therefore, $\alpha$ is less than a constant (depending only on $\delta$ ) times $\theta$. It follows that $d_{\mathbb{E}}\left(0, w_{2}\right) \leq 1 / \sqrt{2}$ for sufficiently small $\theta_{\delta}$.

We conclude that, for $\theta_{\delta}$ sufficiently small and $x>\delta$, the points $w_{1}$ and $w_{2}$ are contained in a ball of radius $1 / \sqrt{2}$, as claimed. Thus,

$$
\begin{aligned}
\operatorname{Vol}_{\mathbb{H}}\left(R_{1}\right) & =\int_{R_{1}} \frac{4 r}{\left(1-r^{2}\right)^{2}} d r d \theta \\
& \leq 16 \int_{R_{1}} r d r d \theta \\
& =16 \operatorname{Vol}_{\mathbb{E}}\left(R_{1}\right) .
\end{aligned}
$$

Observe that $R_{1}$ is contained in the disk with center $1 / x$ and radius $1 / x+1$. In fact, $R_{1}$ is contained in the circular sector of this disk bounded by the ray $\ell$ and the real axis. The latter sector has area $\frac{\theta}{4}\left(1+\frac{1}{x}\right)^{2}$. Hence,

$$
\operatorname{Vol}_{\mathbb{H}}\left(R_{1}\right) \leq C_{\delta}^{\prime \prime \prime} \theta
$$

where $C_{\delta}^{\prime \prime \prime}=\frac{1}{4}\left(1+\frac{1}{\delta}\right)^{2}$.

Combining (16) and (17) proves Claim 3.12 and, therefore, the proposition.

\subsection{Boundary convergence.}

Proposition 3.13. With $G=\mathscr{D}^{\lambda}$ for almost every realization of $G$, the simple random walk started at 0 , considered as a process in the Poincaré disk, converges almost surely in the topology of $\mathbb{C}$ to a point on $S^{1}$.

PROOF. The proof here is a small modification of [13], Theorem 4.1. For any point $z \in \mathbb{C}$, we let $\theta(z)=\frac{z}{|z|}$ be the corresponding point in $S^{1}$. From the hyperbolic law of cosines, we can see that there are absolute constants $c_{1}, c_{2}>0$ so that 
for any two points $p, q \in \mathbb{H}$ with $d_{\mathbb{H}}(p, q) \leq d_{\mathbb{H}}(q, 0)$ with $d_{\mathbb{H}}(q, 0) \geq c_{1}$,

$$
|\theta(p)-\theta(q)| \leq c_{2} e^{-d_{\mathbb{H}}(0, q)+d_{\mathbb{H}}(p, q) / 2} .
$$

By Lemma 1.3 and Borel-Cantelli, we can show that for $G=\mathscr{D}^{\lambda}$

$$
M=\sup _{x \in \Pi^{\lambda}} \max _{\substack{y \in \Pi^{\lambda}, d_{G}(x, y)=1}} \frac{d_{\mathbb{H}}(x, y)}{\log \left(2+d_{\mathbb{H}}(0, x)\right)}<\infty
$$

almost surely. From the almost sure positive speed of $X_{k}$, we have that $d_{\mathbb{H}}\left(X_{k}, 0\right) \geq$ $c^{\prime} k$ for some other $c^{\prime}>0$ and all $k$ sufficiently large. Hence, we get the estimate that

$$
\begin{aligned}
\left|\theta\left(X_{k+1}\right)-\theta\left(X_{k}\right)\right| & \leq \sup _{r>c^{\prime} k} c_{2} e^{-r+M \log (2+r) / 2} \\
& \leq c_{2} e^{-c^{\prime} k+M \log (2+k) / 2}
\end{aligned}
$$

for all $k$ sufficiently large. This is summable in $k$, and hence $\theta\left(X_{k}\right)$ converges almost surely. As $d_{\mathbb{H}}\left(X_{k}, 0\right) \rightarrow \infty$, the proof is complete.

4. Unimodularity of Poisson-Voronoi tilings in symmetric spaces. In this section, we show the Poisson-Voronoi adjacency graph in a Riemannian symmetric space is unimodular. We will give a brief introduction to these spaces. For a more extensive introduction, see [23] or [14], Chapter 10.

A symmetric space $\mathbb{M}$ is a connected Riemannian manifold where at each point $p$, there is an isometry $\sigma_{p}$ of $\mathbb{M}$ that fixes $p$ and whose differential at $p$ is multiplication by -1 . Symmetric spaces are geodesically complete, and hence for any two nonequal points $y, z \in \mathbb{M}$ there is a geodesic $\gamma$ connecting them. On this geodesic, we may find the midpoint $m$ between $y$ and $z$. Then the map $\tau_{y, z}=\sigma_{m}$ interchanges $y$ and $z$ and fixes $m$.

The existence of this symmetry is the reason that the Poisson-Voronoi graph is unimodular. As in the specific case of the hyperbolic Poisson-Voronoi graph, let $\Pi^{\lambda}$ be a Poisson point process with positive intensity $\lambda \cdot d V$ where $\lambda>0$ and $d V$ denotes the volume element on $\mathbb{M}$ (here we will not be concerned about the particular normalization of the volume). We condition $\Pi^{\lambda}$ to have a point at some fixed $x_{0} \in \mathbb{M}$. For any $x \in \Pi^{\lambda}$, let $\mathcal{V}(x)$ denote the Poisson-Voronoi cell with nucleus $x$. Let $\mathscr{G}$ be the dual graph of the Voronoi tessellation, so that two nuclei $x, y \in \mathcal{X}$ are adjacent if and only if there is an open metric ball $B$ with $\{x, y\} \subset \partial B$ and $B \cap \mathcal{X}=\varnothing$.

Riemannian symmetric spaces are prime examples of nonpositively curved spaces, which include, for example, $\mathbb{H}^{d}, \mathbb{H}^{d} \times \mathbb{R}^{k}, \mathbb{H} \times \mathbb{H}$, positive definite matrices, and many others. Strictly speaking, not all Riemannian symmetric spaces are nonpositively curved. However, any Riemannian symmetric space decomposes as a Riemannian product $\mathbb{M}_{1} \times \mathbb{M}_{2}$ where $\mathbb{M}_{1}$ is nonpositively curved and $\mathbb{M}_{2}$ is compact (see [26], Chapter 4, for an overview). Hence, the projection from $\mathbb{M}$ onto $\mathbb{M}_{1}$ 
is a quasi-isometry, so restricting to a nonpositively curved Riemannian symmetric space is natural from the point of view of understanding the large scale geometry of Poisson-Voronoi graphs.

Nonpositive curvature is beneficial for many reasons, one of which is that the notion of convexity translates well to a nonpositively curved space (see [23], Section 1.6). Of particular importance, metric balls are geodesically convex. Note that Voronoi cells, however, will not typically be convex in variable curvature settings (in the Poisson-Voronoi case, they are almost surely never convex). Further, so long as $\mathbb{M}$ is not flat, which is to say that $\mathbb{M}$ is not $\mathbb{R}^{d}, \mathbb{M}$ will have exponential volume growth. In fact, setting $f(r)=\operatorname{Vol}_{\mathbb{M}}\left(B_{\mathbb{M}}\left(x_{0}, r\right)\right)$, we have that there are positive constants $k, h, C$ so that for all $r \geq 1$,

$$
\frac{1}{C}<f(r) r^{-k} e^{-h r}<C
$$

(see [30]). To reiterate, $h=0$ if and only if $\mathbb{M}=\mathbb{R}^{k}$.

Proof of Proposition 1.6. Let $P$ denote the law on $(\mathscr{G}, \rho)$ that arises as the push forward of the point process $\mathcal{X}$, and let $Q$ be the law on rooted graphs with Radon-Nikodym derivative $\frac{d Q}{d P}=\frac{\operatorname{deg} \rho}{\mathbb{E} \operatorname{deg} \rho}$. We continue to use $\mathbb{P}$ to denote the law of the Poisson point process and $\mathbb{E}$ to denote expectation with respect to $\mathbb{P}$.

For any two nonequal points $y, z \in \mathbb{M}$, let $\mathbb{B}_{y, z}$ be the bisector of $y$ and $z$, that is, the submanifold of $\mathbb{M}$ consisting of points that are equidistant from $y$ and $z$, and let $\mathcal{C}_{y, z}$ be the event that $\{y, z\} \subset \mathcal{X}$ and $y$ connects to $z$ in $\mathscr{G}$. For any bijection $\tau: \mathbb{M} \rightarrow \mathbb{M}$, let $\tau^{*}: \sigma(\mathcal{X}) \rightarrow \sigma(\mathcal{X})$ be the induced map on events, that is,

$$
\tau^{*}(\{\mathcal{X} \in V\})=\{\tau(\mathcal{X}) \in V\}
$$

where $V$ is any subset of $\mathbb{M}^{\mathbb{N}}$. As $\mathbb{B}_{y, z}$ is invariant under $\tau_{y, z}$ it follows that $\tau_{y, z}^{*}\left(\mathcal{C}_{y, z}\right)=\mathcal{C}_{y, z}$.

We begin by showing that $\mathbb{E} \operatorname{deg} \rho<\infty$. Recall that $f(r)=\operatorname{Vol}_{\mathbb{M}}\left(B_{\mathbb{M}}\left(x_{0}, r\right)\right)$ satisfies $f(r) \geq c r^{d}$ for some constant $c>0$ and $f(r)<C r^{d} e^{r h}$ for some constant $C>0$. Let $\mathbb{P}_{y}$ be the law of $\mathcal{X}$ conditioned to have points at both $x_{0}$ and $y$. To show that $\mathbb{E} \operatorname{deg} \rho<\infty$, it will suffice to show that

$$
\mathbb{P}_{y}\left[\mathcal{C}_{x_{0}, y}\right] \leq C \exp \left(-f\left(d_{\mathbb{M}}\left(x_{0}, y\right) / 2\right) / C\right)
$$

for some constant $C$, as having shown this it follows that

$$
\begin{aligned}
\mathbb{E} \operatorname{deg} \rho & =\mathbb{E} \sum_{y \in \mathcal{X}} \mathbf{1}\left\{x_{0}, y \text { connected in } \mathscr{G}\right\} \\
& =\lambda \int_{\mathbb{M}} \mathbb{P}_{y}\left(\mathcal{C}_{x_{0}, y}\right) d V(y) \\
& \leq C \lambda \int_{0}^{\infty} \exp (-f(r / 2) / C) f^{\prime}(r) d r .
\end{aligned}
$$

As $f(r / 2) \geq f(r)^{\beta}$ for some $\beta>0$ and all $r \geq 1$, this integral is finite. 
Turning to the proof of (20), recall that $x_{0}$ and $y$ are connected if and only if there is some $u \in \mathbb{B}_{x_{0}, y}$ so that $B_{\mathbb{M}}\left(u, d_{\mathbb{M}}\left(u, x_{0}\right)\right)$ contains no points of $\mathcal{X}$. As balls are geodesically convex in a complete nonpositively curved space (see [23], Section 1.6), this ball also contains the midpoint $m$ of $x_{0}$ and $y$. Hence, $d_{\mathbb{M}}\left(u, x_{0}\right) \geq$ $d_{\mathbb{M}}(u, m)$. Additionally, the bisector is disjoint from $B_{\mathbb{M}}\left(x_{0}, d_{\mathbb{M}}\left(x_{0}, m\right)\right)$ and so $d_{\mathbb{M}}\left(u, x_{0}\right) \geq d_{\mathbb{M}}\left(x_{0}, m\right)$.

Let $\mathcal{U}$ be a maximal 1-separated subset of $\mathbb{B}_{x_{0}, y}$. Note that if $\mathcal{C}_{x_{0}, y}$ occurs, then there is some $u \in \mathcal{U}$ so that $B_{\mathbb{M}}\left(u, d_{\mathbb{M}}\left(u, x_{0}\right)-1\right)$ contains no points of $\mathcal{X}$. Hence, we have the bound

$$
\mathbb{P}_{y}\left[\mathcal{C}_{x_{0}, y}\right] \leq \sum_{u \in \mathcal{U}} \exp \left(-f\left(d_{\mathbb{M}}\left(u, x_{0}\right)-1\right)\right) .
$$

As all the $1 / 2$ balls around $\mathcal{U}$ are disjoint, we have that for any $r>0$

$$
\left|\mathcal{U} \cap B_{\mathbb{M}}(m, r)\right| f(1 / 2) \leq \operatorname{Vol}_{\mathbb{M}}\left(B_{\mathbb{M}}(m, r)\right)=f(r) .
$$

Therefore, we may estimate (21) by partitioning the points into annuli of radius $r-1$ to $r$, which yields

$$
\mathbb{P}_{y}\left[\mathcal{C}_{x_{0}, y}\right] \leq \sum_{r=1}^{\infty} \frac{f(r)}{f(1 / 2)} \exp \left(-f\left(\max \left(d_{\mathbb{M}}\left(x_{0}, m\right), r\right)-2\right)\right) .
$$

It suffices to estimate the sum under the additional assumption that $d_{\mathbb{M}}\left(x_{0}, m\right)>1$ by adjusting constants in (20). Subdivide the sum according to $r<d_{\mathbb{M}}\left(x_{0}, m\right)$ and $r>d_{\mathbb{M}}\left(x_{0}, m\right)$. For $r<d_{\mathbb{M}}\left(x_{0}, m\right)$, we have

$$
\begin{aligned}
& \sum_{r=1}^{\left\lfloor d_{\mathbb{M}}\left(x_{0}, m\right)\right\rfloor} \frac{f(r)}{f(1 / 2)} e^{-f\left(\max \left(d_{\mathbb{M}}\left(x_{0}, m\right), r\right)-2\right)} \\
& \quad \leq d_{\mathbb{M}}\left(x_{0}, m\right) \frac{f\left(d_{\mathbb{M}}\left(x_{0}, m\right)\right)}{f(1 / 2)} e^{-f\left(d_{\mathbb{M}}\left(x_{0}, m\right)-2\right)} .
\end{aligned}
$$

As for $r>d_{\mathbb{M}}\left(x_{0}, m\right)$, recalling that $f(r)$ grows at least polynomially large in $r$, the sum is no more than some absolute constant times its first term. Combining these cases, we get that

$$
\mathbb{P}_{y}\left[\mathcal{C}_{x_{0}, y}\right] \leq C d_{\mathbb{M}}\left(x_{0}, m\right) f\left(d_{\mathbb{M}}\left(x_{0}, m\right)\right) \exp \left(-f\left(d_{\mathbb{M}}\left(x_{0}, m\right)-2\right)\right)
$$

for some absolute constant $C>0$. Applying (19) to the exponent, the desired (20) therefore follows for some other sufficiently large constant $C>0$.

We now turn to the second claim that $\left(\mathscr{G}, X_{0}\right)$ is reversible under the law $Q$. Let $X_{0}=\rho$ and let $X_{1}$ be simple random walk on $\mathscr{G}$ after one step. We will show that

$$
\left(\mathscr{G}, X_{0}, X_{1}\right) \stackrel{\mathcal{L}}{=}\left(\mathscr{G}, X_{1}, X_{0}\right),
$$

as distributions on birooted equivalence classes of random graphs. For this purpose, it suffices to show that for any $r \geq 1$, and any finite rooted graphs $(g, v)$ and $(h, w)$

$$
Q\left[B_{\mathscr{G}}\left(X_{0}, r\right) \cong g \text { and } B_{\mathscr{G}}\left(X_{1}, r\right) \cong h\right]=Q\left[B_{\mathscr{G}}\left(X_{0}, r\right) \cong h \text { and } B_{\mathscr{G}}\left(X_{1}, r\right) \cong g\right],
$$


where $\cong$ denotes equality up to rooted isomorphism. Equivalently, it suffices to show that

$$
\frac{P\left[B_{\mathscr{G}}\left(X_{0}, r\right) \cong g \text { and } B_{\mathscr{G}}\left(X_{1}, r\right) \cong h\right]}{P\left[B_{\mathscr{G}}\left(X_{0}, r\right) \cong h \text { and } B \mathscr{G}\left(X_{1}, r\right) \cong g\right]}=\frac{\operatorname{deg} v}{\operatorname{deg} w} .
$$

For clarity, let $\pi: \mathcal{V}(\mathscr{G}) \rightarrow \mathcal{X}$ be the embedding of the vertices of $\mathscr{G}$ into $\mathbb{M}$. Note that $\pi$ can be taken to be a function of the rooted isomorphism class $(\mathscr{G}, \rho)$. Let $\tau$ be shorthand for $\tau_{x_{0}, \pi\left(X_{1}\right)}$, let $\mathcal{X}^{\prime}=\tau(\mathcal{X})$ and let $\mathscr{G}^{\prime}$ be the dual graph of the Voronoi tessellation with nuclei $\mathcal{X}^{\prime}$. Let $\rho^{\prime}$ be the vertex of $\mathscr{G}^{\prime}$ which is embedded at $x_{0}$. Then $\left(\mathscr{G}^{\prime}, \rho^{\prime}\right)$ is isomorphic to $\left(\mathscr{G}, X_{1}\right)$ as rooted graphs.

Let $\mathcal{E} \in \sigma\left(\mathcal{X}, X_{1}\right)$ be any event on which $\operatorname{deg} \rho$ and $\operatorname{deg} X_{1}$ are both almost surely constants. Denote these by $d_{1}$ and $d_{2}$, respectively. Note that on $\tau^{*}(\mathcal{E})$, $\operatorname{deg} \rho=d_{2}$ and $\operatorname{deg} X_{1}=d_{1}$. By (22), it suffices to show that

$$
d_{2} \mathbb{P}\left[\tau^{*}(\mathcal{E})\right]=d_{1} \mathbb{P}[\mathcal{E}]
$$

Let $\mu$ be the marginal probability measure of $\pi\left(X_{1}\right)$ on $\mathbb{M}$. As the law $\mathcal{X}$ can be viewed as a tight Borel measure on a complete separable metric space (the boundedly finite measures under vague convergence; see [19], Appendix A2.6), we have the existence of a regular conditional probability measure $\mathbb{P}\left[\cdot \mid \pi\left(X_{1}\right)=y\right]$. In particular, we may write

$$
\mathbb{P}[\mathcal{E}]=\int_{\mathbb{M}} \mathbb{P}\left[\mathcal{E} \mid \pi\left(X_{1}\right)=y\right] d \mu(y) .
$$

Let $\mathbb{P}_{y}$ be the law of $\mathcal{X}$ conditioned to have a points at both $x_{0}$ and $y$. Note that for any $y$ we have that $\mathbb{P}_{y}\left[\pi\left(X_{1}\right)=y\right]>0$. Further, by a standard limiting argument, it is easily verified that

$$
\mathbb{P}\left[\mathcal{E} \mid \pi\left(X_{1}\right)=y\right]=\frac{\mathbb{P}_{y}\left[\mathcal{E} \cap\left\{\pi\left(X_{1}\right)=y\right\}\right]}{\mathbb{P}_{y}\left[\pi\left(X_{1}\right)=y\right]}=\mathbb{P}_{y}\left[\mathcal{E} \mid \pi\left(X_{1}\right)=y\right] .
$$

Using that the degrees of $X_{0}$ and $X_{1}$ are specified on $\mathcal{E}$,

$$
\begin{aligned}
\mathbb{P}_{y}\left[\tau^{*}(\mathcal{E}) \cap\left\{\pi\left(X_{1}\right)=y\right\}\right] & =\mathbb{P}_{y}\left[\tau_{x_{0}, y}^{*}(\mathcal{E}) \cap\left\{\pi\left(X_{1}\right)=y\right\}\right] \\
& =\mathbb{P}_{y}\left[\tau_{x_{0}, y}^{*}(\mathcal{E}) \cap \mathcal{C}_{x_{0}, y}\right] \frac{1}{d_{2}}
\end{aligned}
$$

where we have used that $X_{1}$ is simple random walk. As $\tau_{x_{0}, y}$ is an isometry that interchanges $x_{0}$ and $y$, we have $\mathbb{P}_{y} \circ \tau_{x_{0}, y}^{*}=\mathbb{P}_{y}$. As $\mathcal{C}_{x_{0}, y}$ is invariant under $\tau_{x_{0}, y}^{*}$, we have

$$
\begin{aligned}
\mathbb{P}_{y}\left[\tau^{*}(\mathcal{E}) \cap\left\{\pi\left(X_{1}\right)=y\right\}\right] & =\mathbb{P}_{y}\left[\mathcal{E} \cap \mathcal{C}_{x_{0}, y}\right] \frac{1}{d_{2}} \\
& =\mathbb{P}_{y}\left[\mathcal{E} \cap\left\{\pi\left(X_{1}\right)=y\right\}\right] \frac{d_{1}}{d_{2}}
\end{aligned}
$$


Hence, integrating out the conditioning, we have

$$
\begin{aligned}
d_{2} \mathbb{P}\left[\tau^{*}(\mathcal{E})\right] & =\int_{\mathbb{M}} d_{2} \mathbb{P}\left[\tau^{*}(\mathcal{E}) \mid \pi\left(X_{1}\right)=y\right] d \mu(y) \\
& =\int_{\mathbb{M}} d_{1} \mathbb{P}\left[\mathcal{E} \mid \pi\left(X_{1}\right)=y\right] d \mu(y) \\
& =d_{1} \mathbb{P}[\mathcal{E}] .
\end{aligned}
$$

Acknowledgments. This work was largely performed while J. Pfeffer was a participant in Kupcinet-Getz Summer science school at WIS.

We would like to thank Pablo Lessa and Matias Piaggio for sharing an advance version of their work [16] and for pointing out errors in earlier versions of this draft (especially a seriously mistaken claim about the volume growth of $\mathscr{D}^{\lambda}$ ). We also would like to thank Tsachik Gelander for many helpful discussions regarding geometric group theory, and Younghwan Son for many helpful conversations. We also thank the anonymous referees for their careful reading of the manuscript and for helpful suggestions.

\section{REFERENCES}

[1] Ajtai, M., Chvátal, V., Newborn, M. M. and Szemerédi, E. (1982). Crossing-free subgraphs. In Theory and Practice of Combinatorics. North-Holland Math. Stud. 60 912. North-Holland, Amsterdam. MR0806962

[2] Aldous, D. and Lyons, R. (2007). Processes on unimodular random networks. Electron. J. Probab. 12 1454-1508.

[3] Angel, O., Barlow, M. T., Gurel-Gurevich, O. and Nachmias, A. (2016). Boundaries of planar graphs, via circle packings. Ann. Probab. 44 1956-1984. MR3502598

[4] Angel, O., Hutchcroft, T., Nachmias, A. and RAY, G. (2016). Hyperbolic and parabolic unimodular random maps. Preprint. Available at arXiv:1612.08693.

[5] Angel, O., Hutchcroft, T., Nachmias, A. and Ray, G. (2016). Unimodular hyperbolic triangulations: Circle packing and random walk. Invent. Math. 206 229-268. MR3556528

[6] Angel, O., NACHMias, A. and RAY, G. (2016). Random walks on stochastic hyperbolic half planar triangulations. Random Structures Algorithms 49 213-234. MR3536537

[7] Angel, O. and Ray, G. (2015). Classification of half-planar maps. Ann. Probab. 43 13151349. MR3342664

[8] BenJAmini, I. and CURIEN, N. (2012). Ergodic theory on stationary random graphs. Electron. J. Probab. 17 Art. ID 93. MR2994841

[9] Benjamini, I. and Eldan, R. (2012). Convex hulls in the hyperbolic space. Geom. Dedicata 160 365-371. MR2970060

[10] Benjamini, I., Lyons, R., Peres, Y. and Schramm, O. (1999). Group-invariant percolation on graphs. Geom. Funct. Anal. 9 29-66.

[11] Benjamini, I., Lyons, R. and Schramm, O. (1999). Percolation perturbations in potential theory and random walks. In Random Walks and Discrete Potential Theory 56-84. Cambridge Univ. Press, Cambridge.

[12] Benjamini, I. and Schramm, O. (1996). Harmonic functions on planar and almost planar graphs and manifolds, via circle packings. Invent. Math. 126 565-587.

[13] Benjamini, I. and Schramm, O. (2001). Percolation in the hyperbolic plane. J. Amer. Math. Soc. 14 487-507. 
[14] Bridson, M. R. and Haefliger, A. (1999). Metric Spaces of Non-positive Curvature. Grundlehren der Mathematischen Wissenschaften [Fundamental Principles of Mathematical Sciences] 319. Springer, Berlin. MR1744486

[15] Cannon, J. W., Floyd, W. J., Kenyon, R. and Parry, W. R. (1997). Hyperbolic geometry. In Flavors of Geometry. Math. Sci. Res. Inst. Publ. 31 59-115. Cambridge Univ. Press, Cambridge. MR1491098

[16] Carrasco Piaggio, M. and Lessa, P. (2016). Equivalence of zero entropy and the Liouville property for stationary random graphs. Electron. J. Probab. 21 Art. ID 55.

[17] Chen, D. and Peres, Y. (2004). Anchored expansion, percolation and speed. Ann. Probab. 32 2978-2995. MR2094436

[18] Curien, N. (2016). Planar stochastic hyperbolic triangulations. Probab. Theory Related Fields 165 509-540. MR3520011

[19] Daley, D. J. and Vere-Jones, D. (2003). An Introduction to the Theory of Point Processes, Vol. I: Elementary Theory and Methods, 2nd ed. Springer, New York. MR1950431

[20] Dillencourt, M. B. (1990). Realizability of Delaunay triangulations. Inform. Process. Lett. 33 283-287. MR1045517

[21] Dillencourt, M. B. and SMith, W. D. (1996). Graph-theoretical conditions for inscribability and Delaunay realizability. Discrete Math. 161 63-77. MR1420521

[22] Durrett, R. (2010). Probability: Theory and Examples, 4th ed. Cambridge Series in Statistical and Probabilistic Mathematics 31. Cambridge Univ. Press, Cambridge. MR2722836

[23] Eberlein, P. B. (1996). Geometry of Nonpositively Curved Manifolds. Univ. Chicago Press, Chicago, IL. MR1441541

[24] Georgakopoulos, A. (2016). The boundary of a square tiling of a graph coincides with the Poisson boundary. Invent. Math. 203 773-821. MR3461366

[25] Häggström, O., Schonmann, R. H. and Steif, J. E. (2000). The Ising model on diluted graphs and strong amenability. Ann. Probab. 28 1111-1137. MR1797305

[26] Helgason, S. (2001). Differential Geometry, Lie Groups, and Symmetric Spaces. Graduate Studies in Mathematics 34. Amer. Math. Soc., Providence, RI. Corrected reprint of the 1978 original. MR1834454

[27] Kaimanovich, V. A. (1992). Dirichlet norms, capacities and generalized isoperimetric inequalities for Markov operators. Potential Anal. 1 61-82. MR1245225

[28] Kaimanovich, V. A. (1992). Measure-theoretic boundaries of Markov chains, 0-2 laws and entropy. In Harmonic Analysis and Discrete Potential Theory 145-180. Springer, New York.

[29] KARP, L. and Peyerimhoff, N. (2002). Extremal properties of the principal Dirichlet eigenvalue for regular polygons in the hyperbolic plane. Arch. Math. (Basel) 79 223-231. MR1933381

[30] KNIEPER, G. (1997). On the asymptotic geometry of nonpositively curved manifolds. Geom. Funct. Anal. 7 755-782.

[31] Krengel, U. (1985). Ergodic Theorems. de Gruyter Studies in Mathematics 6. de Gruyter, Berlin. MR797411

[32] Lyons, R., Pemantle, R. and Peres, Y. (1995). Ergodic theory on Galton-Watson trees: Speed of random walk and dimension of harmonic measure. Ergodic Theory Dynam. Systems 15 593-619. MR1336708

[33] Lyons, R. and Peres, Y. (2016). Probability on Trees and Networks. Cambridge Univ. Press, New York.

[34] Mohar, B. (1991). The Laplacian spectrum of graphs. In Graph Theory, Combinatorics, and Applications, Vol. 2 (Kalamazoo, MI, 1988) 871-898. Wiley, New York. MR1170831

[35] ThOMASSEN, C. (1992). Isoperimetric inequalities and transient random walks on graphs. Ann. Probab. 20 1592-1600. MR1175279 
[36] VIRÁG, B. (2000). Anchored expansion and random walk. Geom. Funct. Anal. 10 1588-1605. MR1810755

\section{BENJAMINI}

FACULTY OF MATHEMATICS AND

COMPUTER SCIENCE

The WeizMann Institute OF SCIENCE

234 HERZL ST.

REHOVOT 76100

ISRAEL

E-MAIL: itai.benjamini@weizmann.ac.il
E. Paquette

DEPARTMENT OF MATHEMATICS

ThE OHIO STATE UNIVERSITY

100 MATH TOWER

$231 \mathrm{~W} 18 \mathrm{TH}$ AVE.

Columbus, OHio 43210

USA

E-MAIL: paquette.30@osu.edu

J. PFEFFER

DEPARTMENT OF MATHEMATICS

MASSACHUSETtS Institute of TeChNOLOGY

Simons BuILDing (BUILDING 2), Room 106

77 Massachusetts Ave.

CAMbridge, MassachusetTs 02139-4307

USA

E-MAIL: pfeffer@mit.edu 